\title{
A serotonergic axon-cilium synapse drives nuclear signaling to maintain chromatin accessibility
}

Shu-Hsien Sheu ${ }^{1,2,3,4,}{ }^{*}$, Srigokul Upadhyayula5,6, Vincent Dupuy ${ }^{7}$, Song Pang ${ }^{1}$, Andrew L.

5 Lemire$^{1}$, Deepika Walpita ${ }^{1}$, H. Amalia Pasolli,14, Fei Deng ${ }^{8}$, Jinxia Wan ${ }^{8}$, Lihua Wang ${ }^{1}$, Justin Houser ${ }^{9,15}$, Silvia Sanchez-Martinez ${ }^{1,16}$, Sebastian E. Brauchi ${ }^{1,10,11}$, Sambashiva Banala $^{1}$, Melanie Freeman ${ }^{1,17}$, C. Shan $\mathrm{Xu}^{1}$, Tom Kirchhausen ${ }^{9,12,13}$, Harald F. Hess ${ }^{1}$, Luke Lavis ${ }^{1}$, Yu-Long Li ${ }^{8}$, Séverine Chaumont-Dubel ${ }^{7}$, David E. Clapham ${ }^{1,2,4,{ }^{*}}$

1 Janelia Research Campus, Ashburn, VA, USA

102 Harvard Medical School, Boston, MA, USA

${ }^{3}$ Boston Children's Hospital, Department of Pathology, Boston, MA, USA

4 Boston Children's Hospital, Department of Cardiology, Boston, MA, USA

${ }^{5}$ Advanced Bioimaging Center, University of California at Berkeley, Berkeley, CA, USA

${ }^{6}$ Department of Molecular and Cell Biology, University of California at Berkeley,

15 Berkeley, CA, USA

${ }^{7}$ Institut de Génomique Fonctionnelle, Université de Montpellier, CNRS, INSERM,

Montpellier, France

${ }^{8}$ School of Life Sciences, Peking University, Beijing, China

${ }^{9}$ Program in Cellular and Molecular Medicine, Boston Children's Hospital, Boston, MA,

20 USA

${ }^{10}$ Department of Physiology, Faculty of Medicine, Universidad Austral de Chile,

Valdivia, Chile

${ }^{11}$ Millennium Nucleus of Ion Channel-Associated Diseases (MiNICAD), Valdivia, Chile

12 Department of Cell Biology, Harvard Medical School, 200 Longwood Ave, Boston,

25 MA, USA

${ }^{13}$ Department of Pediatrics, Harvard Medical School, 200 Longwood Ave, Boston, MA, USA

${ }^{14}$ current address: The Rockefeller University, New York, NY, USA

${ }^{15}$ current address: Department of Biomedical Engineering, The University of Texas at

30 Austin, Austin, TX, USA

${ }^{16}$ current address: The University of Wyoming, Laramie, WY, USA

17 current address: 10x Genomics, San Francisco, CA, USA

*Correspondence to: sheus@janelia.hhmi.org, claphamd@janelia.hhmi.org 


\section{Summary}

Chemical synapses between axons and dendrites mediate much of the brain's intercellular communication. Here we describe a new kind of synapse - the axo-ciliary synapse - between axons and primary cilia. By employing enhanced focused ion beam -

5 scanning electron microscopy on samples with optimally preserved ultrastructure, we discovered synapses between the serotonergic axons arising from the brainstem, and the primary cilia of hippocampal CA1 pyramidal neurons. Functionally, these cilia are enriched in a ciliary-restricted serotonin receptor, 5-hydroxytryptamine receptor 6 (HTR6), whose mutation is associated with learning and memory defects. Using a newly developed cilia-targeted serotonin sensor, we show that optogenetic stimulation of serotonergic axons results in serotonin release onto cilia. Ciliary HTR6 stimulation

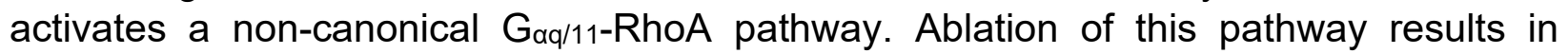
nuclear actin and chromatin accessibility changes in CA1 pyramidal neurons. Axo-ciliary synapses serve as a distinct mechanism for neuromodulators to program neuron transcription through privileged access to the nuclear compartment.

\section{Keywords}

Primary cilia, serotonin, pyramidal neurons, electron microscopy, FIB-SEM imaging, GPCR signaling, fluorescence lifetime imaging, chromatin remodeling, chromatin accessibility, nuclear actin 


\section{Introduction}

The primary cilium is a microtubule-based, membrane-bound compartment that extends a few microns from the basal body into the extracellular space (Bornens, 2012). Ciliopathies, genetic disorders caused by mutant proteins related to cilia function, range from embryonic and perinatal death to situs inversus (left/right reversed visceral organs), polydactyly, kidney cyst formation, obesity, and neurological deficits such as ataxia and intellectual disability. Many of these phenotypes can be attributed to abnormal embryonic development, since the primary cilia house several key components in the Sonic hedgehog (Shh) pathway that orchestrates the processing and release of cilia-housed

10 GLI1-3 transcription factors affecting growth, division, and differentiation (reviewed in Goetz and Anderson, 2010).

Less is known about the normal function of primary cilia in the mature brain, in which most neurons no longer divide or differentiate. Although cilia are lost in most terminally differentiated adult skeletal and cardiac muscle, they are present in most mature neurons and glia of brain (Guemez-Gamboa et al., 2014). There are a few clues that point to their function in adult brain, but most can be interpreted as late onset phenotypes stemming from developmental abnormalities (e.g., Bardet-Biedl syndrome; Barnett et al., 2002). One important fact is that primary cilia in adult brain are enriched in selected G-protein coupled receptors (GPCRs) for neurotransmitters including dopamine, serotonin, norepinephrine, and somatostatin. Indeed, knock-out of a ciliary-localized GPCR, somatostatin receptor 3 (SSTR3), causes novel object recognition cognitive impairment without grossly affecting brain development (Einstein et al., 2010). To gain insight into the potential functions of neuronal primary cilia in the adult brain, we set out to determine how ciliary signaling events are activated in vivo and the sequelae of ligand binding to primary cilia-specific serotonin HTR6 receptors.

\section{Results}

\section{CA1 neuronal cilia have a preferred orientation}

Anti-adenylyl cyclase 3 (ADCY3) antibodies were used to visualize the brain neuronal primary cilia's distribution and orientation in $200 \mu \mathrm{m}$ fixed sections (Bishop et al., 2007;

30 Figure 1A). The preferred trajectory of hippocampal pyramidal neuron's primary cilia is along the basal-apical axis (Figure 1B). This pattern is most striking and uniform in the CA1 region, and least so in the CA2 region. Similar preferred orientations of primary cilia were observed in cortical neurons, which aligned with apical dendrites (Kirschen et al., 2017).

35 We next asked if CA1 cilia preferentially project from the deep hippocampus (basal side of the pyramidal neurons) to the superficial hippocampus (apical side of the pyramidal neurons). If true, this would suggest a possible morphogen gradient along the basalapical axis. We labeled the base of the cilia with an antibody against the ciliary rootlet 
protein, rootletin (CROCC, Figure 1C). Surprisingly, cilia trajectories were largely bidirectional, with about half of cilia projecting to the more superficial layer (stratum radiatum) and the other half projecting to the deeper layer of the hippocampus (stratum oriens, Figure 1D). We hypothesized that cilia trajectory is influenced by special contacts between cilia and nearby structures in the neuropil. To test this hypothesis in mouse brain, we employed volume electron microscopy techniques to visualize neuronal primary cilia and their immediate surroundings.

\section{FIB-SEM reveals axo-ciliary synapses}

We used focused ion beam - scanning electron microscopy (FIB-SEM) to reconstruct the

10 microenvironment of CA1 neuronal primary cilia. In a pilot dataset using $6 \mathrm{~nm} \times 6 \mathrm{~nm} \times$ $20 \mathrm{~nm}$ voxel resolution, we reliably followed two CA1 cilia in a $20 \mu \mathrm{m} \times 20 \mu \mathrm{m} \times 15 \mu \mathrm{m}$ volume (Figure 2A). The most tantalizing observation was that axonal varicosities are often close to CA1 pyramidal neuronal cilia. One striking example is shown in Figure 2A: here the two cilia meet at an axonal bouton containing synaptic vesicles and a mitochondrion, reminiscent of classical presynaptic axonal terminals.

This raised the question of whether pyramidal neuronal cilia are forming specialized contacts with certain axons, and whether these are specialized sites for neurotransmission. We collected eight FIB-SEM datasets of mature mouse hippocampus at $5.5 \mathrm{~nm} \times 5.5 \mathrm{~nm} \times 15 \mathrm{~nm}$ voxel size in $30 \mu \mathrm{m} \times 20-30 \mu \mathrm{m} \times 20-30 \mu \mathrm{m}$ volumes. We

20 found that most cilia have contact sites with axonal processes (80\%, 25 out of 31$)$. Ciliogenesis of pyramidal neurons start around birth and continue to elongate, until finally shortening at 8-12 weeks (Arellano et al., 2012). In addition, developing brains exhibit more extracellular space than mature brains (Lehmenkühler et al., 1993). We hypothesized that axo-ciliary synapses might be more evident in younger brains. Indeed,

25 axo-ciliary synapses are evident in FIB-SEM images of P14 mouse brain (Figure S1, $83 \%, 10$ of 12 samples). In some cases, pyramidal neuronal cilia and axons appear to travel together (Figure S1A). As in adults, there are mitochondria and ER-PM junctions in axonal processes in contact with primary cilia, resembling classic presynaptic boutons (Figure S1B).

30 Next, we tested whether fixation artefacts or simply random coincidence were responsible. High-pressure freezing-freeze substitution (HPF-FS) of live samples better preserves ultrastructure and extracellular space (Hoffman et al., 2020; Korogod et al., 2015; Zechmann et al., 2007). However, it is difficult to preserve samples larger than $10 \mu \mathrm{m}$ without significant formation of ice crystals (Korogod et al., 2015). A hybrid protocol -

35 chemical perfusion followed by HPF-FS provides ultrastructure that is close to direct HPF/FS of live samples for transmission electron microscopy (Sosinsky et al., 2008), but it did not preserve the extracellular space and the contrast was insufficient for FIB-SEM (data not shown). Therefore, we developed a new hybrid protocol to better preserve ultrastructure and extracellular space while providing high contrast for FIB-SEM imaging 40 by employing imidazole and 3-amino,1,2,4 triazole in osmium-based freeze-substitution staining (Figure S2, see Methods). We acquired two isotropic $8 \mathrm{~nm} \times 8 \mathrm{~nm} \times 8 \mathrm{~nm}$ FIB- 
SEM datasets of adult mouse CA1 samples prepared with the hybrid protocol using enhanced FIB-SEM systems ( $35 \mu \mathrm{m} \times 35 \mu \mathrm{m} \times 40 \mu \mathrm{m}$ and $50 \mu \mathrm{m} \times 50 \mu \mathrm{m} \times 44 \mu \mathrm{m})$. In these two datasets, we identified a total of 27 neuronal primary cilia and similar putative axonal-primary cilia contact sites, or axo-ciliary synapses, in 18 out of 27 neuronal cilia (67\%, Figure 2C-E, Video S1). These synapses are characterized by a $20-40 \mathrm{~nm}$ cleft between the axon and the cilium, flanked by areas in which the axonal membrane and ciliary membrane are immediately in apposition (Figure 2C). In axonal varicosities, synaptic vesicles can be seen within $20 \mathrm{~nm}$ of the axonal plasma membrane, or occasionally, appear to be docking or fusing with the plasma membrane, suggestive of vesicular release (Figure $\mathbf{2 C}$, red arrows). Also seen in the axon are endoplasmic reticulum forming junctions with the plasma membrane (ER-PM junctions) and mitochondria, as seen in classical presynaptic terminals (Wu et al., 2017; Figure 2D-E). Interestingly, contrast enhancement is seen at the ciliary membrane next to the axonal varicosity (Figure 2C, green arrow). These features resemble classical chemical 15 synapses.

\section{Axo-ciliary synapses are serotonergic}

Since the axons that form axo-ciliary synapses originate from neurons outside the FIBSEM datasets, we sought to determine the identity of these axons. HTR6, a serotonin G protein-coupled receptor, is highly enriched in neuronal primary cilia (Brodsky and Neumaier, 2017). We hypothesized that the axons in contact with cilia are serotonergic axons that innervate the cilia through the HTR6 receptor. By using an endogenous Htr6EGFP knock-in mouse line (Nadim et al., 2016), we first confirmed that HTR6 is restricted to pyramidal neuronal primary cilia (Figure 3A). Thirty-five percent (426 out of 1209) of cilia are in close apposition to serotonergic axons (Figure 3B-C), as measured by colabeling cilia and the serotonin transporter (SERT, SLC6A4, a marker for serotonergic axons) using super-resolution Airyscan confocal microscopy. In addition, all axonal sites opposing cilia contain synaptophysin staining, suggesting that these are serotoninrelease sites (Figure 3D-E; Belmer et al., 2017). Since the apposition distance could be subject to variables such as antibody accessibility and optical chromatic aberrations (see Methods), we examined the distribution of the shortest distance to the central axes of serotonergic axons (skeletonized axons) among all ciliary voxels (central axes) and all cilia central axes (skeletonized cilia, Figure 3F-G). Both showed a skewed distribution towards short distances, suggesting that ciliary trajectories are biased towards serotonergic axons. In addition, since ligand stimulation is known to result in ciliary remodeling, including ciliary ectocytosis, decapitation, withdrawal, or shedding (Mirvis et al., 2019; Nager et al., 2017), our analysis of a single snapshot in time might underestimate the frequency of axo-ciliary synapses.

We noticed that the axon-contacting cilia are slightly longer than the non-contacting cilia (Figure 3H; median length 7.0 versus $6.4 \mu \mathrm{m}, p<0.0001$, two-tailed Mann-Whitney $U$ test). However, this length difference of $<1 \mu \mathrm{m}$ is unlikely to explain why some cilia are in contact with serotonergic axons while others are not. Indeed, we failed to detect any 
significant correlation between cilia length and the shortest distance to serotonergic axons (Figure 3G, Pearson correlation coefficient $r=-0.19$ ), suggesting that simply increasing ciliary length would not result in an increased likelihood of contacts with serotonergic axons. Interestingly, contacting cilia exhibit greater variance in length (standard deviation: $3.3 \mu \mathrm{m}$ for contacting cilia, versus $3.0 \mu \mathrm{m}$ for non-contacting cilia; two-group distribution comparison $\mathrm{p}$-value with permutation test $<0.0001$ ), this implies that serotonergic axoncontacting cilia may receive higher levels of stimulation and thus have more frequent changes in length. Altogether, these results suggest that CA1 pyramidal neuronal cilia receive serotonergic innervation from the raphe nuclei (Muzerelle et al., 2016).

\section{Activation of serotonergic axons releases serotonin onto cilia}

The ultrastructural and super-resolution analyses above provide anatomical evidence of axo-ciliary synapses. Next, we sought to determine if serotonin is released onto cilia upon the activation of serotonergic axons. Fluorescent reporters often do not traffic to cilia (Delling et al., 2013), so we first fused an extracellular serotonin sensor (Unger et al., 2020) onto the N-terminus of HTR6. Unfortunately, the HTR6 receptor was aberrantly targeted. As an alternative, we engineered a ciliary-targeted serotonin sensor based on the GPCR-activation-based (GRAB) strategy with the HTR6 receptor as the scaffold (GRAB-HTR6-PM; Wan et al., 2021). We first expressed this sensor in HEK-293T cells, which trafficked well to membranes with an $\sim 150 \%$ fluorescence increase in response to saturating 5-HT (EC50 = 84 nM; Figure S3; human HTR6 receptor $\mathrm{K}_{\mathrm{D}}=37 \mathrm{nM}$; Monsma et al., 1993). Therefore, we reasoned that this sensor could detect ciliary serotonin changes at physiologically relevant levels. We then removed the lgK leader sequence and added a HaloTag on the C-terminus to better visualize cilia with bright Janelia Fluor dyes (Grimm et al., 2015; Zheng et al., 2019). This resulted in robust cilia targeting in RPE-1 cells and neurons, as when HTR6 is expressed (Figure 4A). The EC50 for the cilia-targeted HTR6-GRAB-cilia sensor is $28 \mathrm{nM}$, with up to $40 \%$ fluorescence increase per cilium in response to saturating doses of 5-HT using 3D Airyscan (Figure 4B). The smaller increase in maximum fluorescence of the cilia-targeted sensor (40\% versus $150 \%$ ) may be attributed to a different lipid composition of the ciliary membrane (reviewed in Conduit and Vanhaesebroeck, 2020) and/or the increased bleaching due to laser scanning/Z-stack imaging required to 3-dimensionally image single cilia.

We attempted imaging ciliary serotonin dynamics in acute hippocampal slices with mice injected with adeno-associated virus (AAV) carrying the sensor construct. However, we could not achieve good signal-to-noise ratios in deeper areas $(>20 \mu \mathrm{m})$, in which surface damage can be avoided with either Airyscan or lattice light-sheet microscope with adaptive optics (data not shown). Therefore, we developed an in-vitro system of serotonergic axo-ciliary synapses by co-culturing hippocampal and serotonergic neurons from the midbrain (Figure S4). We expressed the cilia-serotonin sensor GRAB-HTR6cilia and ChrimsonR, a red-shifted channelrhodopsin (Klapoetke et al., 2014), in

40 hippocampal and serotonergic neurons, respectively. Using Airyscan, we detected serotonin release onto cilia that were in synaptic contact with serotonergic axons (average 
peak $\Delta F / F=0.15$, Figure 4C-D; Figure S5). This increase is diminished in nonchannelrhodopsin controls (mean difference, or effect size by estimation statistics $=-0.10$, $95 \% \mathrm{Cl}=-0.14$ to -0.7 , permutation test $\mathrm{p}$-value $=0.0002$, two-tailed Mann-Whitney test $\mathrm{p}$-value $=0.0009$, Figure $\mathbf{4 C , E}$; Figure S5). Interestingly, the serotonin release is attenuated on cilia distant from serotonergic axons (mean difference, or effect size by estimation statistics $=-0.09,95 \% \mathrm{Cl}=-0.14$ to -0.05 , permutation test $p$-value $=0.005$, two-tailed Mann-Whitney test p-value $=0.008$, Figure 4C,F; Figure S5). Although the differences are near the limit of detection due to the sensitivity of the sensors/detectors and the small volumes being measured, the data suggest that firing of serotonergic axons results in serotonin release on hippocampal neuronal cilia. Future experiments may be possible using 5HT3 channel-containing sniffer pipettes placed in apposition to the releasing membrane to detect serotonin, although surface accessibility currently limits such approaches.

\section{Serotonin stimulation activates a neuronal ciliary HTR6-Gaq/11-Trio-RhoA pathway}

15 To examine the functional significance of the axo-ciliary synapses, we studied serotonininduced HTR6 activation on cilia. HTR6 is characterized as a Gas-coupled GPCR, activating adenylyl cyclase to increase cAMP when over-expressed on the plasma membrane of dividing HEK cells (Boess et al., 1997). However, HTR6 activation does not increase cAMP when it is localized in cultured cell primary cilia (Jiang et al., 2019).

20 However, GPCRs may interact with multiple G-proteins (Flock et al., 2017; Masuho et al., 2015; Okashah et al., 2019). Indeed, GPCR-G protein coupling differs in some ciliated vs non-ciliated cells (Masyuk et al., 2013) and the same GPCR might be coupled to different Ga-subunits (Hilgendorf et al., 2019) on the plasma membrane vs the cilia. Therefore, we hypothesized that cilia-localized HTR6 interacts with a different $\mathrm{G}_{\alpha}$ subunit. The $\mathrm{G}_{a 11-}$ subunit (GNA11) was previously identified as a binding partner of the endogenous HTR6 in the brain through affinity purification and mass spectrometry (Nadim et al., 2016), contrasting with AP-MS data obtained by heterologous expression of HTR6 in dividing HEK cells, which identified the $\mathrm{G}_{\text {as }}$-subunit (GNAS), as a binding partner (Meffre et al., 2012).

30 Gaq/11 can also activate the Trio-RhoA pathway in C. elegans and in Gaq/11-constitutively active mutant uveal melanoma cells, as determined through a forward genetic screen and a genome-wide siRNA screen, respectively (Feng et al., 2014; Williams et al., 2007). Trio was identified in the HTR6-ciliome (Kohli et al., 2017) and is detected in cilia by immunofluorescence in HTR6-HaloTag RPE-1 cells and in WT cultured hippocampal

35 neurons (Figure S6A). Indeed, serotonin activates RhoA in HTR6-overexpressing HEK cells and in neurons in which the receptor is distributed throughout the plasma membrane and neuronal processes, suggesting that HTR6 may signal through the Gaq/11-Trio-RhoA pathway (Rahman et al., 2017). We thus hypothesized that neuronal ciliary HTR6 expressed at the endogenous levels might activate RhoA through $\mathrm{G}_{\mathrm{aq} / 11-}$ Trio.

40 To our knowledge, RhoA activity has not been directly measured in primary cilia. We first tested several translocation based RhoA sensors with fluorescent proteins fused to a 
protein domain that binds to active RhoA (Mahlandt et al., 2021). However, these sensors caused either significant cell death or formed cytoplasmic aggregates in neurons (data not shown). We then tested FRET-based RhoA sensors, in which a pair of fluorescent proteins is fused to RhoA and a protein domain that binds to active RhoA, respectively. We targeted a FRET-based RhoA sensor (Bindels et al., 2017) to the cilia by fusing it to HTR6 and expressed it in RPE-1 cells (Figure S6C). To better recapitulate the serotonin release at the axo-ciliary synapses, we considered employing caged serotonin next to the cilium using UV-releasable (N)-1-(2-nitrophenyl) ethyl (NPEC)-caged serotonin (Breitinger et al., 2000). To avoid damaging UV radiation, we synthesized a new photoactivatable, caged serotonin molecule that can be cleaved by $405 \mathrm{~nm}$ laser light (Figure S6B). Caged serotonin stimulation $(0.5 \mathrm{~Hz})$ immediately adjacent to the cilium elicited a pulsatile increase in RhoA activity, returning to near baseline upon cessation (Figure S6C-E). However, uncaging suffered from a high failure rate and the average FRET ratios across cilium can be significantly affected by just a few pixels due to the 15 small size of cilium.

We sought to obtain better measurements of ciliary RhoA by fluorescence-lifetime measurements (FLIM/FRET) to minimize the effect from donor bleaching and better account for the difference in sensor levels. In this measurement, we expect the donor lifetime to decrease from FRET. As cilia often span multiple Z-levels while FLIM is normally carried out without optical sectioning, we first tested whether FLIM with optical sectioning across the $Z$ axis can be achieved by using a fast FLIM system equipped with a pulsed white light laser (Rolf et al., 2021). We were able to reconstruct whole HEK293A cells with HTR6-RhoA sensor expression through FLIM imaging (Figure 5A). The Arl13bRhoA sensor is functional in cilia since stimulation by a RhoA activator (Flatau et al., 1997; Schmidt et al., 1997) decreased the fluorescence lifetime of the donor significantly (the effect size, or mean difference by estimation statistics $=-60 p s, 95 \% \mathrm{Cl}=-100 p s$ to $-24 p s$, permutation test $p$-value $=0$, two tailed Mann-Whitney test $p$-value $=0.002$, Figure 5BC). HTR6-RhoA cilia have higher RhoA activity than Arl13b-RhoA cilia, suggesting that over-expression of HTR6 results in constitutive activity (mean difference, or effect size by estimation statistics $=-125 p s, 95 \% \mathrm{Cl}=-162 p s$ to $-93 p s$, permutation test $p$-value $=0$, Mann-Whitney test p-value $<0.00001$, Figure 5D-E), as commonly seen in GPCR signaling (reviewed in Seifert and Wenzel-Seifert, 2002).

We then measured serotonin-dependent RhoA activity in neuronal cilia. We expressed the Arl13b-RhoA sensor, as cultured hippocampal neurons have ciliary HTR6 (Figure 5F). Due to instrument limitations, we were unable to perform photo-uncaging concurrently with FLIM measurements. Instead, we used a low concentration of $5 \mathrm{HT}$ (10 nM; rat receptor $\mathrm{K}_{\mathrm{D}}=12.7 \mathrm{nM}$; Boess et al., 1997) to minimize receptor desensitization and better recapitulate the pulsed nature of serotonin release by axonal firing. Ten nanomolar $5 \mathrm{HT}$ stimulation reliably increased RhoA activity in neuronal cilia in 5 to 15 minutes (the effect size, or the estimation statistics $=-63 p s, 95 \% \mathrm{Cl}=-106 p s$ to $-41 p s$, permutation test $p-$ value $=0$, Wilcoxon $p$-value $=0.00001$, Figure 5G-H). Adding a HTR6 blocker SB258585 (100 nM, Hirst et al., 2000) 5 min before 10 nM 5HT application largely abolished this 
effect (Figure $\mathbf{5 H}$ ), suggesting that the increase in RhoA requires HTR6. We then asked if the increase required $G_{\alpha q / 11}$. Pretreatment with a $G_{\alpha q / 11}$ inhibitor, $Y M-254890(1 \mu M$; Nishimura et al., 2010; Takasaki et al., 2004) mitigated the RhoA increase in neuronal cilia (Figure 5 H). Gaq/11 KO HEK293A cells have significantly lower ciliary RhoA activity; Figure 5D-E). Lastly, pre-treatment with YM-254890 abolished RhoA spikes seen in RPE-1 cells (Figure S6D-E). Together, these data suggest that serotonin stimulation results in $\mathrm{G}_{a \mathrm{q} / 11}$-dependent RhoA activation in cilia.

Next, we tested ciliary RhoA activity upon chemogenetic activation of serotonergic axons in the hippocampal neuron - raphe neuron co-culture system. We expressed the Arl13bRhoA sensor and an excitatory Designer Receptors Exclusively Activated by Designer Drugs (DREADD) hM3Dq (Armbruster et al., 2007) in hippocampal neurons and raphe neurons, respectively. Application of $10 \mathrm{nM} \mathrm{hM3Dq}$ DREADD agonist deschloroclozapine (DCZ, Nagai et al., 2020) increased RhoA activity in cilia that apposed serotonergic axons within 5 min (Figure 5I-K). In some cases, we observed ciliary withdrawal and/or retraction, or receptor retrieval from the cilia. In contrast, there was no detectable increase in RhoA activity in non-contacting cilia (Figure 5K). This suggests that the ciliary RhoA activation is under spatial and temporal control of the activity of serotonergic axons.

\section{Nuclear actin-associated changes and decreased chromatin accessibility after ciliary HTR6 ablation}

20 RhoA activation is known to induce cytoplasmic F-actin polymerization and myosin activation (reviewed in Hodge and Ridley, 2016). As cilia originate from the neuronal cell somata, we hypothesized that ciliary RhoA signaling can affect somatic actin-related structures. The most notable examples of actin-related structures in neuronal cell somata are actin lattices revealed by dSTORM imaging (Han et al., 2017). These lattices were

25 revealed using antibodies against the actin binding protein, adducin, and resemble the classic lattices seen in red blood cells (Bennett and Gilligan, 1993; Pan et al., 2018). RhoA activation can phosphorylate adducin through Rho-associated kinase and increase its affinity towards F-actin (Fukata et al., 1999). Consistent with Han et al. (2017), we detected adducin plasma membrane labeling in neuronal cell somata (Figure 6A). When

30 we treated cultured hippocampal neurons with the HTR6 antagonist SB-742457 (Upton et al., 2008), however, we did not see significant changes in plasma membrane adducin, but nuclear adducin was enriched in a small subset of neurons (Figure 6B). This change is reminiscent of nuclear translocation of adducin reported in cultured cells that affect cell growth and division (Chen et al., 2011; Liu et al., 2017).

35 As cells grown on hard surfaces such as glass can alter actin dynamics, we examined adducin staining patterns in the native hippocampal environment. Surprisingly, we did not detect plasma membrane adducin staining in the neuronal cell somata in the hippocampus, but pyramidal neurons exhibit variable numbers of clear nuclear adducin puncta (Figure 6C). In Htr6 knockout (KO) mice pyramidal neurons, the density of

40 adducin puncta increased significantly (Figure 6D, effect size, or mean difference by estimation statistics within $5 \mu \mathrm{m}$ radius from the center of nuclei $=25.5,95 \% \mathrm{Cl}=20.4$ to 
47.6, permutation test $p$-value $=0$, two-tailed Mann-Whitney test $p$-value $<0.0001$, consistent with our HTR6 antagonist experiments. This suggests that ciliary HTR6 signaling is linked to nuclear actin.

Alterations in nuclear actin modify global chromatin (Plessner and Grosse, 2019; Zhao et al., 1998). Increased nuclear actin by disruption of cytoplasmic actin can alter cellular state to drive differentiation (Sen et al., 2017), while reducing nuclear actin can result in cell quiescence (Spencer et al., 2010). To test chromatin remodeling directly in the brain, we employed ATAC-see, a technique that utilizes a hyperactive transposase mutant with fluorescently labeled oligonucleotides (Chen et al., 2016; Xie et al., 2020). The transposase has high reactivity only to open chromatin regions, resulting in fluorescent labeling of accessible chromatin areas in cells. We performed ATAC-see with superresolution Airyscan on age- and gender-matched samples from WT and Htr6 KO adult mice. In Htr6 KO CA1 samples, the labeling intensity was significant less than the intensity in WT samples (the effect size, or median difference by estimation statistics $=-108,95 \%$ $\mathrm{Cl}=-128$ to -77 , permutation test $\mathrm{p}$-value $=0$, two tailed Mann-Whitney test $p$-value $<$ 0.0001 , Figure 6E-F). This change in ATAC-see labeling is reminiscent of the difference between early and late phase G1 cells, in which cells decondense their chromatin after mitosis (Chen et al., 2016). We conclude that ablation of the HTR6 receptor results in altered patterns of accessible chromatin.

20 To further test if chromatin changes were indeed ciliary RhoA-dependent, we expressed a cilia targeted TrioRhoGEF inhibitor peptide (Bouquier et al., 2009) under a tetracycline inducible promoter. In adult mice fed for 1-week with doxycycline containing food, there was notable neuronal loss with pyknotic nuclei (Figure 6G). The remaining, comparatively healthier neurons had decreased ATAC-see labeling intensity compared to nondoxycycline food treated mice (the effect size, or median difference by estimation statistics $=-244,95 \% \mathrm{Cl}=-259$ to -225 , permutation test $\mathrm{p}$-value $=0$, two tailed MannWhitney test $p$-value $<0.0001$, Figure $6 \mathbf{G}-\mathrm{H}$ ). Taking all our results together, the most parsimonious conclusion is that primary ciliary HTR6 signaling controls chromatin

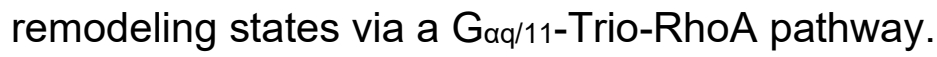

30 Htr6 KO is associated with loss of accessibility of cis-regulatory elements of genes related to learning and memory

To further characterize the chromatin accessibility changes in Htr6 KO mice, we utilized single-cell ATAC sequencing. We performed nuclei isolation, tagmentation, and sequencing in WT and Htr6 KO hippocampus, followed by pooled data processing

35 (Figure 7A-B). We observed 19 unique cell clusters in pooled WT and KO nuclei (Figure 7C). Cell types were assigned based on differentially enriched marker peaks and known gene expression profiles of the mouse hippocampus (Figure S7 and Table S1, see Methods; Zeisel et al, 2018). CA1 neurons were identified as clusters 1, 9, and 13 using GPR161 and Ndst3 as marker genes. Interestingly, the difference between WT and Htr6 40 KO CA1 neurons are readily apparent in pooled clustering. While cluster 9 is only in WT neurons $(n=622)$, cluster 1 is mostly present in the KO sample $(n=1652$ and $n=117$ in KO 
and WT, respectively, Figure 7D). We compared accessible loci between cluster 1 (KO) and 9 (WT), between all WT CA1 and KO CA1 neurons, and between WT and KO cluster 13. Consistent with our data from ATAC-see, there is significant net loss of accessible loci in all three comparisons in KO neurons (Figure 7E). For all CA1 neurons, KO cells have 3340 loci with lower accessibility than WT, while having 1111 loci with higher accessibility levels.

Most of the less accessible loci are distant to transcription start sites (TSS), which may be long range enhancer elements $(40 \%$ being $>50 \mathrm{~kb}$ upstream of TSS, $27 \%$ being $>50$ $\mathrm{kb}$ downstream of TSS, with only $9 \%$ within $\pm 5 \mathrm{~kb}$ of TSS sites). In contrast, $40 \%$ of more accessible loci are within $\pm 5 \mathrm{~kb}$ of TSS, or around the promoter area (Figure $7 \mathrm{~F}$ ). This pattern is strikingly similar to that found between low and hi-G1 cells (Chen et al, 2016). The pattern suggests that the ciliary HTR6 signaling axis in mature neurons might impact transcription primarily through long-range enhancers, such as the prototypic Shh enhancer that controls the expression of Shh gene during development (reviewed in Schoenfelder and Fraser, 2019), Gli repressors through altering enhancer activities (Li et al., 2016), or ciliary FFAR4 receptor-driven adipogenesis through CTCF-binding enhancers (Hilgendorf et al., 2019).

Finally, we sought to determine genes that are impacted by loss of Htr6. By using nearby genes of the less accessible loci ( $n=5829$, GREAT analysis, McLean et al., 2010), we performed Gene Ontology (Ashburner et al., 2000; The Gene Ontology Consortium et al., 2020) and Reactome (Jassal et al., 2019) term enrichment analyses using g:Profiler (Raudvere et al., 2019). The significantly enriched terms include cell-cell junction and communication, Gaq signaling pathways, heparan sulfate and heparin (HS-GAG) biosynthesis and metabolism, cell projection, and synapses (Figure 7G). Notably, a recent study showed that depleting cilia completely in CA1 neurons through IFT88 shRNA knock-down results in defective extracellular matrix/perineuronal net and memory impairment (Jovasevic et al., 2021), suggesting a potential convergent path for these different perturbations. Together, these changes corroborate the synaptic and cognitive deficits reported in Htr6 KO mice (Sun et al., 2021).

\section{Discussion}

We presented evidence of synapses between raphe serotonin-secreting axonal neurons and HTR6 serotonin receptor-expressing primary cilia of CA1 pyramidal neurons. We 1) identified axo-ciliary synapse structures; 2) identified primary cilia HTR6 receptors adjacent to serotonergic axonal varicosities containing vesicles and other markers of synapses; and 3) demonstrated serotonin release onto cilia upon optogenetic stimulation of serotonergic axons. We provided evidence that ciliary HTR6 can activate the noncanonical $\mathrm{G}_{\mathrm{aq} / 11-T r i o-R h o A}$ pathway in primary cilia. Abrogation of this pathway in mature neurons alters nuclear actin via adducin translocation, thus modulating hippocampal function by altering chromatin accessibility and transcriptional pathways. 
Since free serotonin levels in the murine hippocampus are $\sim 300 \mathrm{fM}$ (Schechter et al., 2007) while the $K_{D}$ for HTR6 binding to serotonin is $12.6 \mathrm{nM}$ (Boess et al., 1997), the axociliary synapses provide a mechanism to localize and concentrate serotonin's effect. The ciliary HTR6-Trio-RhoA signaling axis limits serotonin-RhoA signaling to the cilium and exploits its specialized link to the nucleus, much as the Shh pathway regulating Gli transcription factors is limited by compartmentalized $\mathrm{G}_{\text {as }} / \mathrm{PKA}$ signaling. This rationalizes the persistence of primary cilia in non-dividing mature cells such as neurons and can explain how alterations in ciliary signaling can impact structures such as excitatory synapses on dendrites that can be hundreds of microns distant (Tereshko et al., 2021). Interestingly, in other cells such as pre-adipocytes, omega-3 fatty acids were shown to activate primary ciliary FFAR4 receptors to induce CTCF-dependent chromatin changes through opening of CTCF-binding enhancer sites (Hilgendorf, 2019). This posits a tantalizing theme in which primary cilia act as an epigenetic regulator to stabilize transcriptional programming in response to environmental cues. In this "cilia as the

15 nuclear antenna" model, cilia provide a shorter and more direct spatial pathway for neurotransmitters and other receptor agonists to specifically regulate nuclear transcription.

Not all cilia form serotonergic axo-ciliary synapses. This raises the question of whether the neuron hosts of these cilia are specialized among CA1 pyramidal neurons. Perhaps the non-serotonergic synapsing cilia are enriched in other GPCRs (Dopamine receptor 1 (DRD1), somatostatin 3 (SSTR3), galanin receptors (Hilgendorf et al., 2016) or tyrosine kinase receptors that forms axo-ciliary synapses with a different set of axons and elicit different functional networks. The fact that the portion of cilia with axo-ciliary synapses detected in FIB-SEM $(\sim 67 \%$ to $80 \%)$ is greater than those found with HTR6-serotonergic

25 axons (35\%) supports this possibility. Further studies examining different ciliary receptor and axon types will address these questions.

\section{Ciliary signaling and chromatin remodeling}

It is not currently clear how the loss of serotonin mediated ciliary RhoA activation results in changes in nuclear actin and chromatin accessibility. Presumably, activated RhoA may

30 directly traffic to the nucleus, but ciliary RhoA activation may activate Rho-associated kinases (ROCK1 and ROCK2), which subsequently localize to the nucleus. Nuclear ROCK2 has been shown to phosphorylate EP300, or histone acetyltransferase p300, that can regulate transcription via chromatin opening (Tanaka et al., 2006). Our current data suggests that serotonin signaling in adult primary cilia maintains one state, and its

35 removal initiates a change leading to adducin/actin mediated chromatin remodeling.

The time variation of physiological changes in brain serotonin provokes other questions. The raphe serotonergic system is much more active in wake states than during sleep (Oikonomou et al., 2019; Wan et al., 2021). Interestingly, the transcript levels of HTR6 also oscillates and peaks around midnight, or before the serotonergic system becomes

40 active (Baldi et al., 2021). We do not know if HTR6 axo-ciliary synapses oscillate and may be involved in chromatin remodeling during sleep/wake cycles (Hor et al., 2019) which 
may impact learning and memory (Rasch and Born, 2013). A recent genome-wide association study identified HTR6 as one of the 15 genes indicated in bipolar disorders (Mullins et al., 2021), while a somatic HTR6 mutation was detected in resected cortical seizure foci with dysplastic growth (Zhang et al., 2020). In addition, HTR6 is also expressed in upper and lower motor neurons, another class of pyramidal neurons (Bandyopadhyay et al., 2013). Interestingly, in zebrafish, serotonergic inputs to the motor neurons promote adult regeneration of motor neurons (Barreiro-Iglesias et al., 2015). It will be interesting to determine if serotonergic axo-ciliary synapse and HTR6-RhoA driven chromatin maintenance are also present in these neurons, which may provide mechanistic insight into their survival.

The major importance of this work is the discovery of a new kind of chemical synapse, one that conveys information more directly to the target cell's nucleus. A remaining question is whether the axon-cilia-nucleus axis is a general mechanism for maintaining a stable state in neurons and other cells, as loss of this function results in major chromatin reorganization. The current work suggests that loss of ciliary HTR6 RhoA activation results in nuclear actin changes and alterations in chromatin accessibility. Finally, does disruption of these primary cilia-dependent stable connections lead to programmatic functional changes, resulting in changes in memory or, for example, differentiation status? More broadly does disruption of primary cilia-specific receptors contribute to cancer, type Il diabetes, and other disease states in brain and other organs?

\section{Acknowledgements}

We thank members of the Clapham Lab, Lavis Lab, Lippincott-Schwartz Lab, and the Marin Lab for productive discussions and critical reading of the manuscript (Drs. William Valinsky and Alex Miller). We thank Liangqi (Frank) Xie (Tjian and Liu Lab) for kindly providing the Tn5 transposase and Tn5 transpose-ATTO-590-oligonucleotide mixture solution. We thank Dr. Joachim Goedhart (University of Amsterdam) and Dr. Kees Jalink (Netherlands Cancer Institute) for input on our RhoA-FRET and RhoA-FLIM analyses, respectively. We thank Jeffrey Marshman (Zeiss) for help with the Zeiss Crossbeam FIBSEM. We thank the Janelia Anatomy and Histology, Cell and Tissue Culture, Vivarium,

30 Molecular Biology Shared Resources, and iEplore-Biocampus animal facility for their support. Funding: This work was supported by the Howard Hughes Medical Institute. The generation of Htr6-EGFP knock-in mice and Htr6 KO mice were funded by the Foundation for Medical Research (FRM, France), and two ANR contracts Sero6Cognet (ANR-11-BSV4-008), and Sero6Dev (ANR -17-CE16-0010-01). V.D. was supported by

35 the French ministry of research and education. S.H.S. was supported by NIH 5T32HL110852-05. S.E.B. is supported by ANID-Millennium Science Initiative Program \#NC160011. S.U. was supported by Philomathia Foundation and Chan Zuckerberg Initiative Imaging Scientist program. T.K. was supported by National Institute of General Medical Sciences Maximizing Investigators' Research Award GM130386; National Institutes of Health R01 GM075252; Biogen Sponsored Research Agreement. 


\section{Author Contributions}

Conceptualization, S.H.S. and D.E.C.; Methodology, S.H.S., S.E.B., S.B., C.S.X, F.D., J. W., Y.L.L, J.H., T.K., A.L.L.; Investigation, S.H.S., G.U., V.D., S.E.B., S.B., A.L.L., L.W., F.D., J.W., Y.L.L, M.F., H.A.P., S.P., C.S.X., T.K.; Writing - Original Draft, S.H.S.; Writing

5 -Review \& Editing, S.H.S., L.L., S.E.B., S.C.D., D.E.C.; Funding Acquisition, S.H.S., L.L., S.C.D., T.K., and D.E.C.; Resources, H.F.H.; Visualization, S.H.S., G.U., A.L.L.; Supervision, S.H.S. and D.E.C.

\section{Declaration of Interests}

Portions of the technology described herein are covered by U.S. Patent 10,600,615

10 titled "Enhanced FIB-SEM systems for large-volume 3D imaging", which was issued to C.S.X., K.J.H., and H.F.H., and assigned to Howard Hughes Medical Institute on March 24,2020 . The other authors declare no competing interests. 


\section{Figure Legends}

Figure 1. Adult hippocampal pyramidal neuronal cilia are oriented. (A) Hippocampal coronal $200 \mu \mathrm{m}$-thick section maximum intensity projection (MIP). Green: cilia (ADCY3), red: CA2 (PCP4), blue: nuclei (Hoechst 33342). (B) Orientation (structure tensor) analyses of cilia voxels in CA1, CA2, and CA3. $100 \mu \mathrm{m}$ maximum intensity projection, showing cilia oriented along the basal-apical axis (stratum oriens - stratum radiatum). The images are rotated such that the basal-apical axes are at a $90^{\circ}$ angle. Top panel: Color survey of cilia voxels encoded by orientation (hue), coherence (saturation), and fluorescence intensity (brightness). Bottom panel: normalized weighted frequency distribution with basal-apical axes at $90^{\circ}$, showing original data (blue) and fitted gaussian curves (orange). Mean values of the gaussian distributions are $106^{\circ}, 106^{\circ}$, and $66^{\circ}$ for CA1, CA2, and CA3, respectively. The tails of CA2 fit less well than for CA1 and CA3, indicating more heterogeneity in CA2 cilia vectors. (C) Labeling of ciliary base. CA1 cilia (green) and Rootletin (magenta; CROCC, ciliary rootlet); nuclei (blue, Hoechst 33342);

$1550 \mu \mathrm{m}$ maximum intensity projection. (D) Two cilia oriented at $180^{\circ}$ in $\mathrm{C}$ are magnified. 5 $\mu \mathrm{m}$ maximum intensity projection.

Figure 2. FIB-SEM reveals axo-ciliary synapses. (A) Rendering of a $15 \mu \mathrm{m} \times 15 \mu \mathrm{m} \times$ $10 \mu \mathrm{m}$ dataset imaged at $6 \mathrm{~nm} \times 6 \mathrm{~nm} \times 20 \mathrm{~nm}$ resolution. Two complete cilia (yellow and blue) arise from the basal bodies, which are surrounded by Golgi-related vesicles and Golgi stacks (pink). Note that the axonal varicosity (green) contains a mitochondrion (lavender) and synaptic vesicles (white, arrow) at the crossing point of the two cilia. Yellow arrows: the portions of the cilia that have identifiable microtubule doublets $(2-3 \mu \mathrm{m}$; colored in saturated yellow and blue, respectively). (B) Primary cilia have a $9+0$ microtubule configuration and become $9+1$ more distally. No identifiable microtubule doublets are observed in the most distal $(6-8 \mu \mathrm{m})$ segments (average diameter $100 \mathrm{~nm}$ ). (C) Selected single EM sections of axo-ciliary synapses. Cilium: yellow; axon: cyan asterisk. Top left: An oblique section reconstructed from the volume in (A) to show the longitudinal cross section of the cilium. Note that in some areas, the cilium (yellow) and axonal membrane are in direct contact. Occasional vesicles can be seen within 10-20 nm of the axonal membrane opposing the cilium (red arrow). Bottom left: enhanced contrast at the ciliary membrane next to the axon from volume in A (green arrow). Distance between cilia membrane and axonal membrane at this section is $\sim 20 \mathrm{~nm}$. Top right and bottom right: selected examples with features suggestive of vesicular docking/fusion at the axonal plasma membrane apposing the cilium from dataset rendered in D-E (red arrow). (D) Rendering from an $8 \mathrm{~nm} \times 8 \mathrm{~nm} \times 8 \mathrm{~nm}$ isotropic FIB-SEM dataset. An axonal process (cyan) gives rise to a varicosity (white box) that makes direct contact with a pyramidal neuron primary cilium (yellow). White box is magnified in the right panel. Synaptic vesicles in white, endoplasmic reticulum in red, mitochondrion in green. (E) Another example of axo-ciliary synapse in the same FIB-SEM dataset. A pyramidal neuronal primary cilium (yellow) originates from the left (base not shown) and makes a contact with an axonal varicosity (blue). The area marked by the white arrow is magnified in the right panel. Synaptic vesicles are rendered as $40 \mathrm{~nm}$ spheres to facilitate visualization (white). Note the axonal ensheathment of the cilium, and the proximity of the 
vesicles to the axonal plasma membrane apposing primary cilia. Endoplasmic reticulum: red, mitochondrion: green.

Figure 3. 5-hydroxytryptamine (5-HT; serotonin) receptor (HTR6)-expressing primary cilia are in contact with serotonergic axonal varicosities. (A) HTR6

5 (endogenously tagged with EGFP, amplified with anti-GFP antibody and Alexa 488, green in the merged panel) is highly enriched in CA1 neuronal primary cilia (adenylyl cyclase type 3 immunostaining: ADCY3, magenta in the merged panel). (B) ADCY3-cilia (magenta) are aligned with serotonergic axons (immunostained with anti-serotonin transporter antibody: SERT, SLC6A4, green). Both neuronal primary cilia and serotonergic axons lie along the basal-apical axis; $20 \mu \mathrm{m}$ maximum intensity projection. (C) cilia in B, color-coded with shortest distance to a serotonergic axon. (D) cilia in B, color-coded with shortest distance to a serotonergic axon-associated synaptophysin punctum. (E) magnified white box area from $B$, showing a cilium contacting serotonergic axonal synaptophysin varicosities. Serotonergic axon, synaptophysin, and ADCY3 (cilia) are colored in green, yellow, and magenta in the merged panel, respectively. (F) Density plot showing the relationship between cilium length and the shortest distance to a serotonergic axon on a per voxel basis (cilium central axis). (G) Density plot showing the relationship between cilia length and shortest distance to a serotonergic axon per cilium (central axis). Notice the lack of a linear correlation, and the skewed distribution towards shorter distances. $(\mathbf{H})$ Violin plots showing the distribution of ciliary length of serotonergic axon-contacting and non-contacting cilia. Red circle: median; black bar: $95 \%$ confidence interval of the median. The difference in the shapes of the violin plots and the length of the $95 \%$ bars reflect the greater variance of contacting cilia.

Figure 4. Activation of serotonergic axons releases serotonin onto cilia. (A) RPE-1 cells stably expressing a Tet-inducible HTR6-GRAB-cilia-HaloTag serotonin sensor. 100 nM application results in increased GFP fluorescence. HaloTag:JF552 is used to reliably identify and segment cilia. (B) Titration curve of the sensor. (C) Statistical analyses of ciliary serotonin levels with optogenetic stimulation of serotonergic axons, shown in the Cumming estimation plot. The raw data is plotted on the upper axes. On the lower axes, mean differences are plotted as bootstrap sampling distributions. Each mean difference is depicted as a dot. Each $95 \%$ confidence interval is indicated by the ends of the vertical error bars. Peak $\Delta F / F$ is calculated by filtering the averaged measurements using a $1 \mu \mathrm{m}$ circle at the contact sites (ChrimsonR and non-ChrimsonR contacting cilia controls), or the location on the cilium that is closest to a serotonergic axon (ChrimsonR noncontacting cilia) using a low pass filter (see Figure S5 and Methods). (D-F) Top: A cilium expressing the HTR6-GRAB-cilia sensor in contact with ChrimsonR-tdTomatoexpressing serotonergic axons (D), in contact with a SNAP:JF552 labeled serotonergic axon (non-channelrhodopsin control, E), and a cilium distant from a ChrimsonRtdTomato-expressing serotonergic axon (F). Bottom: color maps showing $\Delta \mathrm{F} / \mathrm{F}$ during optogenetics stimulation (after 25 pulses at $1 \mathrm{~Hz}$ ), corresponding to the 3 different examples in the top row, respectively. 
Figure. 5. Serotonin stimulation of ciliary HTR6 activates RhoA in cilia. (A) HEK293A cells stably expressing the HTR6-RhoA FRET/FLIM sensor. A single cilium arises from the dome-shaped cell soma (FLIM). (B-C) Cilia-targeted Arl13b-RhoA sensor responds to RhoA activation. The mean difference between control and CNO3- (RhoA activator) treated cells is shown in the Gardner-Altman estimation plot. Both groups are plotted on the left axes; the mean difference is plotted on a floating axis on the right as a bootstrap sampling distribution. The mean difference is depicted as a dot; the $95 \%$ confidence interval is indicated by the ends of the vertical error bar. (D-E) GNAQ/11 KO and HTR6overexpression decreases and increases RhoA activity, respectively. The mean differences for 2 comparisons against the shared control Arl13b WT are shown in the Cumming estimation plot. The raw data is plotted on the upper axes. On the lower axes, mean differences are plotted as bootstrap sampling distributions. Each mean difference is depicted as a dot. Each $95 \%$ confidence interval is indicated by the ends of the vertical error bars. (F-H) $10 \mathrm{nM} 5 \mathrm{HT}$ stimulation of neuronal cilia increases ciliary RhoA activity.

15 This effect is blocked by either HTR6 blocker SB258585 (100 nM) or the $\mathrm{G}_{\mathrm{aq} / 11}$ blocker YM-254890 (1 $\mu \mathrm{M})$. (I-K) Chemogenetic stimulation of serotonergic axons increases ciliary RhoA activity in contacting cilia but not in non-contacting cilia. Arrow in I points to area magnified in the inset, which is shown at an oblique angle to demonstrate the close apposition of axon and cilium at the synapse. Contrast is enhanced in the 10 min time

20 point in $\mathrm{J}$. For $\mathrm{H}$ and $\mathrm{K}$, the paired mean differences for comparisons are shown in the Cumming estimation plot. The raw data is plotted on the upper axes; each paired set of observations is connected by a line. On the lower axes, each paired mean difference is plotted as a bootstrap sampling distribution. Mean differences are depicted as dots; $95 \%$ confidence intervals are indicated by the ends of the vertical error bars.

Figure 6. Modulation of the HTR6 signaling axis alters adducin localization and chromatin accessibility. (A-B) DIV28 hippocampal neurons were treated either with $0.01 \%$ DMSO control (A), or $100 \mathrm{nM} \mathrm{SB}-742457$ (B) for 20 min. In DMSO-treated neurons, adducin is primarily at the plasma membrane. In contrast, in some SB-742457-treated neurons, there is significant nuclear labeling. Pyramidal neurons are identified by MAP2 labeling in both cases. Color scheme in merged panels: Blue: Hoechst 33342, green: adducin, red: MAP2. Cilia are colored in magenta (ADCY3 staining, arrow) in the merged maximum intensity projection (MIP) of the entire neuron, while other panels are single optical sections through the middle of the nucleus. (C) Htr6 KO mice exhibit increased numbers of pyramidal neurons with nuclear adducin (ADD1) puncta. (D) Heatmap of number of ADD1 nuclear puncta (color-coded) using the center of nucleus (represented in rows) with increasing distance from 1 to $8 \mu \mathrm{m}$ (represented in columns). There is increased density of puncta in KO cells. (E) Representative single optical Airyscan section of CA1 pyramidal neurons showing ATAC-see labeling with ATTO-590 dye (segmented with a nuclear mask, see Methods). The labeling is significantly decreased in the KO mice, 40 which is quantified in (F). (G) Representative single optical Airyscan section of CA1 pyramidal neurons showing ATAC-see labeling with ATTO-565 dye (segmented with a nuclear mask, see Methods). The labeling is significantly decreased in the mice fed with doxycycline-containing food to induce the expression of the cilia-targeted TrioRhoGEF 
inhibitor. Notice the decreased cell density and small, pyknotic nuclei (arrow). The difference between nuclei that are $>8 \mu \mathrm{m}$ in diameter (not judged pyknotic) is quantified in the estimation statistics plot $(\mathbf{H})$. For $F$ and $H$, the median difference between the two groups is shown in the Gardner-Altman estimation plot. Both groups are plotted on the left axes; the median difference is plotted on a floating axis on the right as a bootstrap sampling distribution. The median difference is depicted as a dot; the $95 \%$ confidence interval is indicated by the ends of the vertical error bar.

Figure 7. Single-cell ATAC sequencing reveals loss of accessibility to long-range enhancer elements and activity changes in genes associated with synapses and the perineuronal net. (A) Overall workflow. (B) Pooled clustering among all nuclei (KO and WT). (C) All 19 detected clusters were merged into defined hippocampal cell types based on marker peaks (see Methods). (D) WT CA1 (cluster 9) and KO CA1 (cluster 1) are enriched in different clusters (pooled). The number of nuclei for cluster 13 increased in KO. (E) Analyses of differential peaks between KO and WT, showing on average a 3fold greater number of less accessible vs more accessible loci. (F) Less accessible loci in $\mathrm{KO}$ are mostly distant from transcription start sites (TSS; red), while more accessible loci are primarily near promoters (gray). (G) Gene ontology and Reactome terms enriched in genes affected by the lost loci. REAC: Reactome; GO: Gene Ontology; BP: biological process.; CC: cellular components.

\section{Supplementary Figure Legends}

Figure S1. FIB-SEM reconstruction of P14 (juvenile) CA1 pyramidal neuron. Related to Figure 2. The neuronal cilium fasciculates with an axonal process. Like adult pyramidal neuronal cilia, axo-ciliary synapses are apparent. Yellow: cilium, cyan: axon, bright green: basal body, red: axonal endoplasmic reticulum, green: axonal mitochondria.

25 Figure S2. Hybrid fixation protocol preserves extracellular space. Related to Figure 2. In contrast to conventional glutaraldehyde perfusion protocols (Kasthuri et al., 2015; right, orange box magnified in the inset with yellow border), the novel hybrid protocol preserves the extracellular space (orange box magnified in the inset with yellow border). Note the rounded morphology of neuronal processes and significantly greater amount of

30 extracellular space (blue). See Methods for details.

Figure S3. The amino acid sequence and characterization of the GRAB-HTR6-PM sensor. Related to Figure 4. (A) Schematic diagram of GRAB-HTR6-PM sensor's structure. The cpEGFP and linkers were transplanted from GRABNE. (B) The amino acid sequence of the GRAB-HTR6-PM sensor. The numbering used in the figure starts from the IgK leader sequence. Insertion sites Q263 ${ }^{\text {ICL3 }}$ and $\mathrm{H} 576^{6.26}$ are indicated by black arrowheads below relevant amino acids. (C) Representative images show the expression of GRAB-HTR6-PM sensor (left, before application of $10 \mu \mathrm{M} 5-\mathrm{HT}$, middle, after application of $10 \mu \mathrm{M} 5-\mathrm{HT}$ ) and the response (right, pseudocolor) in HEK293T cells. Scale bar, $20 \mu \mathrm{m}$. (D) The dose-response curve of the HTR6-GRAB-PM sensor tested in 
HEK293T cells; EC50 labeled. Data are shown as mean \pm S.E.M. and $n=3$ wells with 300-500 cells per well.

Figure S4. Serotonergic axo-ciliary synapse in vitro. Related to Figure 4. (A) Overall workflow. Hippocampal and raphe neurons were dissociated from the hippocampus and midbrain, respectively. In some experiments, constructs were electroporated separately before co-plating in the same well (see Methods). (B) Serotonergic axo-ciliary synapses occur in vitro. On average (1 million cell total, 300,000/cm $\mathrm{cm}^{2}$ density, $1: 1$ hippocampal and midbrain cell ratio), there are 5-10 serotonergic neurons and $\sim 5$ axo-ciliary synapses per well.

10 Figure S5. Measurement of GRAB-HTR6-cilia of axo-ciliary synapses. Related to Figure 4. Fluorescence intensity changes during optogenetic stimulation (starting at $20 \mathrm{~s}$ ) across three different groups. Blue: raw trace, orange: raw trace filtered by a low pass filter $(0.2 \mathrm{~Hz})$. The peaks of the filtered traces during photostimulation were used for statistical testing shown in Figure 4C.

15 Figure S6. Ciliary Gaq-Trio-RhoA signaling in RPE-1 cells. Related to Figure 5. (A)Trio is present in HTR6-cilia in RPE-1 cells and WT cultured hippocampal neuronal cilia. Top panel: RPE-1 cells stably expressing the Tet-inducible HTR6-HaloTag. HaloTag was labeled with Janelia Fluor 552 (magenta in the merged panel), fixed, and stained with an antibody against the Trio GEF-D2 domain (green, merged panel). Lower panel: Trio is

20 present in WT cultured hippocampal neuronal cilia. DIV28 cultured rat hippocampal neurons were fixed and immunostained with anti-ADCY3 antibody (neuronal cilia marker, magenta; merged panel), anti-MAP2 antibody (neuronal marker, red; merged panel), Hoechst 33342 (nucleus, blue; merged panel), and anti-Trio GEF-D2 antibody (green, merged panel). The Trio GEF-D2 signal in both cases was amplified with the Alexa 488

25 tyramide signal amplification system (Thermo Fisher Scientific). Images were processed with the Subtract Background (50 pixels with sliding paraboloid) algorithm in ImageJ/Fiji to enhance contrast for qualitative demonstrations. (B) Properties of photo-activatable ("caged") serotonin (PA-Ser). Top left: Chemical structure of PA-Ser. Top right: Absolute absorption spectrum of a solution of PA-Ser $(10 \mu \mathrm{M})$ in PBS. This molecule displayed an

30 absorption maximum of $380 \mathrm{~nm}$ with an extinction coefficient $(\varepsilon)$ of $21,100 \mathrm{M}^{-1} \mathrm{~cm}^{-1}$; the relatively broad absorption spectrum gives substantial absorption at $405 \mathrm{~nm}(\varepsilon=12,100$ $\mathrm{M}^{-1} \mathrm{~cm}^{-1}$ ). Upon photolysis, PA-Ser releases $\sim 10 \%$ of serotonin along with other major photoproducts generated primarily via a photo-Claisen pathway (Wong et al., 2017). Lower panel: Synthesis of PA-Ser through alkylation of Boc-protected serotonin (S1) with

35 \{7-[bis(carboxymethyl)amino] coumarin-4-yl\} methyl (BCMACM) bromide (S2). This coumarin-based BCMACM photolabile group exhibits high aqueous solubility and relatively large one- and two-photon action cross-sections (Hagen et al., 2008). (C-E) Serotonin stimulation of ciliary HTR6 activates RhoA. RPE-1 cells stably expressing a Tet-inducible HTR6-RhoA FRET based sensor. C. Donor emission (sGFP2), sensitized 40 emission ( $m$ Scarlet-I), and FRET ratio calculated by dividing sensitized emission by donor emission of a single cilium. Local serotonin uncaging at $0.5 \mathrm{~Hz}$ results in RhoA activity 
spikes (D Top panel shows a sample trace, quantified in $\mathbf{E}$ top panel, $p$ value=0.04). The effect is largely attenuated by pre-treating samples with the Gaq/11 blocker, YM-254890 ( $1 \mu \mathrm{M}, \mathbf{D}$ middle panel is a sample trace, quantified in $\mathbf{E}$ middle panel, $p$ value=0.12). Mock uncaging had minimal effect on the RhoA FRET ratio ( $D$ lower panel is a sample trace, quantified in $\mathbf{E}$ lower panel, $\mathrm{p}$ value $=0.52$ ). For $\mathbf{E}$, the spikes are defined as $\Delta \mathrm{F} / \mathrm{F}$ greater than or equal to 0.52 (the mean \pm 3 S.D. in the $5-\mathrm{HT}$ uncaging measurements). Horizontal lines represent the median values. Statistical tests comparing before, during, and after uncaging used the Friedman test (non-parametric).

Figure S7. Marker genes for cell type assignment. Related to Figure 7. For each individual marker gene, we plotted the average gene activity level (see Methods) and percent of cells that expresses the gene. Top panel: original clusters; bottom panel: merged clusters.

Graphical Abstract. Model of the serotonergic axo-ciliary synapse. Identification of synapses between serotonergic axons and pyramidal neuron primary cilia. Cilia-specific serotonin receptor constitutes a preferred signaling pathway to the nucleus. Loss of this pathway results in chromatin remodeling.

Table S1. Number of WT and KO cells in each individual cluster and marker genes. Related to Figure 7.

Video S1. Reconstruction of CA1 neuronal cilia. Related to Figure 2. Movie showing

20 the reconstructed CA1 pyramidal neuronal primary cilia using 8-nm isotropic FIB-SEM imaging. 


\section{STAR METHODS}

\section{RESOURCE AVAILABILITY}

\section{Lead contact}

Further information and requests for resources and reagents should be directed to and will be fulfilled by the lead contact, David Clapham (claphamd@janelia.hhmi.org).

\section{Materials availability}

All unique/stable reagents generated in this study are available from the lead contact. Sharing of the RPE-1 cells are limited by terms set by ATCC.

\section{Data and code availability}

10 scATAC-seq datasets have been deposited to Gene Expression Omnibus (accession number GSE183431).

Any additional information required to reanalyze the data reported in this paper is available from the lead contact upon request.

\section{EXPERIMENTAL MODEL AND SUBJECT DETAILS}

\section{Animals}

All animal work was approved by the Boston Children's Hospital Institutional Animal Care and Use Committee (IACUC 16-03-3138R), the Janelia Institutional Animal Care and Use Committee (IACUC 16-146 and 19-181), or the animal use and care guidelines of Montpellier University (France, authorization D34-172-4). C57BL/6 mice were obtained from Charles River Laboratories. Htr6-EGFP knock-in mice were generated at the Institut Clinique de la Souris (Illkirch-Graffenstaden, France). Htr6 KO mice were generated at the Phenomin consortium (Institut Clinique de la Souris, Illkirch-Graffenstaden, France) by using CRISPR-Cas9. Htr6 exon 3 and 4 were targeted using two pairs of guide RNAs on each side of the targeted region. No restrictions were imposed on food and water. For doxycycline induction experiments, mice were fed with doxycycline-containing food (2000 ppm, Animal Specialties and Provisions, modified from LabDiet 5053) for 1 week. Similar results were obtained in both males and females.

\section{Cell culture}

RPE-1 (female) and HEK293A cells (gift from Dr. Asuka Inoue, Tohoku University, Japan) were plated at $\sim 20,000$ cells $/ \mathrm{cm}^{2}$ on \#1.5 $12 \mathrm{~mm}$ coverslips in 24-wells, 1-chamber 35 $\mathrm{mm}$ glass-bottom dishes, or 4-chamber $35 \mathrm{~mm}$ glass-bottom dishes (all \#1.5 cover glass, Cellvis) in 10\% serum containing media (RPE-1: DMEM:F12 media, ATCC 30-2006; HEK293A: DMEM, low glucose, GlutaMax, pyruvate, Thermo Fisher Scientific \#10567014; Day 0). With HEK293A cells, the dishes were coated with Matrigel (Corning

35 Life Sciences) before plating. The next day (Day 1), the cells were serum deprived with $0 \%$ FBS media with $100 \mathrm{ng} / \mathrm{ml}$ doxycycline to induce GRAB-HTR6-cilia, HTR6-RhoA, or Arl13b-RhoA expression. For HEK393A cells, $1 \mu \mathrm{M} \mathrm{H}-89$ was also added to induce 
ciliogenesis. After 24 h of serum deprivation (Day 2), GRAB-HTR6-cilia cells were labeled with $250 \mathrm{nM}$ Janelia Fluor 552 (JF552) dye for $2 \mathrm{~h}$. Cells were rinsed and placed back in serum-free media without doxycycline or H-89. Experiments were conducted at 48 to 96 $\mathrm{h}$ after serum deprivation (Day 3 to 5 ).

5 For stable cell line creation, hTERT RPE-1 cells (ATCC CRL-4000) or HEK293A cells were transfected with the piggyBac hyperactive transposase vector (VectorBuilder) and HTR6-RhoA sensor vector or GRAB-HTR6-cilia concurrently with Lipofectomine 3000 (Thermo Fisher Scientific) at a 1:2.5 ratio and grown in 10\% Tet-free FBS (Gemini) containing media. The cells were then selected by blasticidin at $10 \mu \mathrm{g} / \mathrm{ml}$ to create Teton HT6-RhoA sensor stable cells.

\section{Primary hippocampal and raphe neuron culture}

Hippocampi and midbrains were dissected from P0 Sprague-Dawley rat pups of both sexes. Rat maintenance and care followed policies advocated by NRC and PHS publications and approved by the Institutional Animal Care and Use Committee (IACUC), Janelia Research Campus. Tissues were digested with papain and gently triturated and filtered through a $40 \mu \mathrm{m}$ filter. Neurons were electroporated (Lonza 4D-nucleofactor) with Arl13b-RhoA (hippocampal neurons) or Tph2-Cre (tryptophan hydroxylase 2-Cre, midbrain neurons) and plated in poly-D-lysine coated dishes and cultured in NbActive medium (Brainbits) at $37^{\circ} \mathrm{C}$ and $5 \% \mathrm{CO}_{2}$. A week after plating, the neuronal cultures were

20 fed with B-27 plus neuronal culture system (Thermo Fisher Scientific). AAV transduction (FLEX-on hM3DGq-DREADD, FLEX-on farnesylated SNAP, or FLEX-on tdTomato) were applied at DIV10. $100 \mathrm{ng} / \mathrm{ml}$ doxycycline was added at DIV 6 or DIV14 and removed the next day. Images were collected between DIV 9 and DIV 28 (AIr13b-RhoA, hippocampal culture only) or DIV21-35 (hippocampal and midbrain co-culture). JF552-STL (SNAPlabeled neurons) was applied the day prior to imaging.

\section{METHOD DETAILS}

\section{Synthesis of PA-0-5-hydroxytryptamine (photoactivatable serotonin)}

$\mathrm{N}$-Boc serotonin (S1, $60 \mathrm{mg}, 217 \mu \mathrm{mol}, 3.5 \mathrm{eq}$ ) and coumarin bromide (S2, $30 \mathrm{mg}, 62.2$ $\mu \mathrm{mol}, 1 \mathrm{eq}$ ) were dissolved in $\mathrm{CH}_{3} \mathrm{CN}(4 \mathrm{~mL}) . \mathrm{K}_{2} \mathrm{CO}_{3}$ (potassium carbonate, $60 \mathrm{mg}, 435$ $\mu \mathrm{mol}, 2 \mathrm{eq}$ ) was added and the reaction was stirred at room temperature for $15 \mathrm{~h}$. The reaction was concentrated under reduced pressure and the residue was dissolved in EtOAc. This was washed with water and saturated $\mathrm{NaCl}(\mathrm{aq})$, dried over $\mathrm{MgSO}_{4}$, and concentrated under reduced pressure. The material was purified using flash chromatography on silica gel (0-50\% EtOAc/hexanes, linear gradient), which afforded 35 $\mathrm{mg}(83 \%)$ of compound $\mathbf{S 3}$ as a pale-yellow solid. Compound $\mathbf{S 3}$ (30 mg, $44.3 \mu \mathrm{mol}$ ) was dissolved in $\mathrm{CH}_{2} \mathrm{Cl}_{2}(2 \mathrm{~mL})$. Trifluoroacetic acid (TFA; $0.4 \mathrm{~mL}$ ) was added and the reaction was stirred at room temperature for $2 \mathrm{~h}$ while shielded from light. Toluene $(5 \mathrm{~mL})$ was added and the mixture was concentrated under reduced pressure. The residue was purified by reverse-phase HPLC using a gradient of $\mathrm{CH}_{3} \mathrm{CN} / \mathrm{H}_{2} \mathrm{O}$ containing $0.1 \% \mathrm{v} / \mathrm{v}$ TFA as additive. 1H NMR (400 MHz, 1:1 CD3OD, CD3CN) $\delta 7.71$ (s, 1H), 7.64 (d, J = 8.9 Hz, 
$1 \mathrm{H}), 7.31(\mathrm{~d}, \mathrm{~J}=8.9 \mathrm{~Hz}, 1 \mathrm{H}), 7.16(\mathrm{~d}, \mathrm{~J}=2.4 \mathrm{~Hz}, 1 \mathrm{H}), 7.13(\mathrm{~s}, 1 \mathrm{H}), 6.93(\mathrm{dd}, \mathrm{J}=8.8,2.5$ $\mathrm{Hz}, 1 \mathrm{H}), 6.65(\mathrm{dd}, \mathrm{J}=9.0,2.7 \mathrm{~Hz}, 1 \mathrm{H}), 6.52(\mathrm{~d}, \mathrm{~J}=2.6 \mathrm{~Hz}, 1 \mathrm{H}), 6.32(\mathrm{~s}, 1 \mathrm{H}), 5.30(\mathrm{~s}, 2 \mathrm{H})$, $4.24(\mathrm{~s}, 4 \mathrm{H}), 3.15(\mathrm{t}, \mathrm{J}=7.3 \mathrm{~Hz}, 2 \mathrm{H}), 3.01(\mathrm{t}, \mathrm{J}=7.3 \mathrm{~Hz}, 2 \mathrm{H})$. HRMS (ESI) calculated for $\mathrm{C}_{24} \mathrm{H}_{24} \mathrm{~N}_{3} \mathrm{O}_{7}[\mathrm{M}+\mathrm{H}]+466.1609$, was 466.1615.

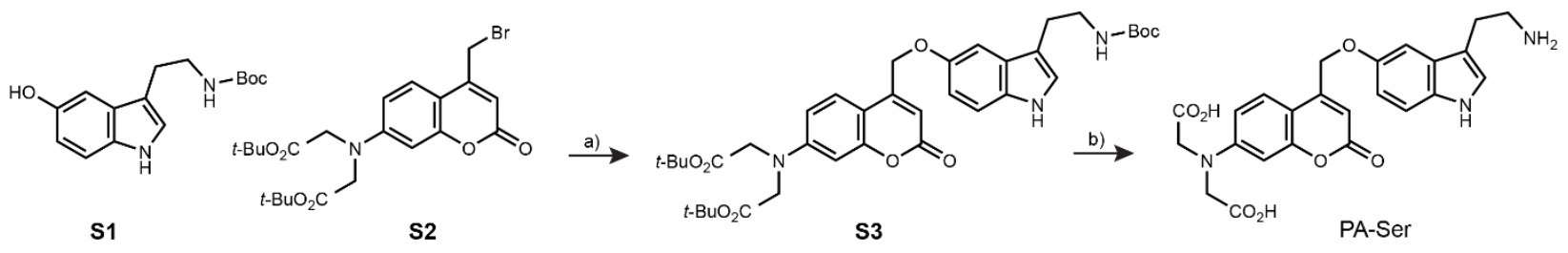

\section{Molecular Biology}

The piggyBac Tet-On HTR6-RhoA sensor was generated based on the published mScarlet-I based RhoA sensor (Bindels et al, 2016). The mScarlet-l:sGFP2:RhoA and the cpPKN1 fragments were synthesized (Genscript) and cloned into piggyBac Tet-On vector Xlone HTR6-HaloTag (Deo et al., 2019). The Xlone-Ar13b-RhoA sensor was subcloned by replacing HTR6 with ARL13B (VectorBuilder). GRAB-HTR6-cilia was made by removing the IgG leader sequence and adding a HaloTag to the $\mathrm{C}$-terminus based on GRAB-HTR6-PM (see Figure S3 and below) by VectorBuilder. Tph2-Cre vector was generated by replacing GFP in Tph2-GFP (Wan et al, 2016; gift from Dr. Björklund) with the Cre-recombinase in Syn1-EBFP-Cre AAV (gift from Hongkui Zeng, Addgene plasmid \# 51507; RRID:Addgene_51507). pAAV-Syn-FLEX-rc[ChrimsonR-tdTomato] was a gift from Edward Boyden (Addgene plasmid \# 62723; RRID:Addgene_62723). pAAV[FLEXON]-CAG-Farnesylated-SNAPtag AAV plasmid was made by VectorBuilder, Inc. pAAVFLEX-ON-tdTomato was a gift from Edward Boyden (Addgene plasmid \# 28306; 20 RRID:Addgene_28306). pAAV-EF1a-DIO-hM3D(Gq)-mCherry was a gift from Bryan Roth (Addgene plasmid \# 50460; RRID:Addgene_50460). pAAV-TRE-HTR6-SNAPfTRIP and pAAV-Syn-Tet3G were made by VectorBuilder. Syn-Tet3G and TRE-HTR6SNAPf-TRIP were packaged in AAV2/rh10 capsid, and the other viral vectors were packaged in the AAV2/PHP.eB capsid. All viral vectors were made by the HHMI Viral 25 Tools.

\section{Intracranial injections}

6-week-old adult mice were anesthetized with 2.5\%-3.0\% isoflurane with an $\mathrm{O}_{2}$ rate of $1 \mathrm{~L} / \mathrm{min}$ and mounted on a stereotaxic frame. The body temperature was maintained at $37^{\circ} \mathrm{C}$ using a heating pad. Mice were given buprenorphine $(0.1 \mathrm{mg} / \mathrm{kg})$ subcutaneously at $5 \mathrm{mg} / \mathrm{kg}$ of body weight. After shaving, a drill was used to create a small craniotomy hole $(\sim 1 \mathrm{~mm}) .200 \mathrm{~nL}$ of AAV mixture composed of AAV-rh10-Syn-Tet3G and AAV-rh10-TREHTR6-TRIP (1:2 ratio, $5 \times 10^{12} \mathrm{vg} / \mathrm{ml}$ and $10^{13} \mathrm{vg} / \mathrm{ml}$, respectively) were bilaterally injected in the mouse CA1 pyramidal neuron layer (anteroposterior $-2.25 \mathrm{~mm}$ relative to Bregma, mediolateral $\pm 2 \mathrm{~mm}$ relative to Bregma; dorsoventral $-1.43 \mathrm{~mm}$ relative to the pial surface) at a rate of $50 \mathrm{nl} /$ minute using a Nanoject II Injector (Drummond Scientific, USA) followed by 5 additional minutes to allow diffusion. Upon recovery, mice were given Ketaprofen 
(5mg/kg, sub-cutaneous) and placed back in the home cage. Ketoprofen $(5 \mathrm{mg} / \mathrm{kg}$, subcutaneous) was given once a day for an additional two days post-surgery.

\section{Immunofluorescence and Imaging}

For cultured cells, \#1.5 coverslips were first fixed in 4\% paraformaldehyde (PFA) in PBS

5 overnight at $4^{\circ} \mathrm{C}$. Samples were then rinsed in PBS for $5 \mathrm{~min} \times 3$. After permeabilization with $0.3 \%$ Triton-X in PBS for 30 min, samples were blocked with $5 \%$ normal goat serum (NGS) in PBS for $1 \mathrm{~h}$. 5\% NGS was then replaced with primary antibody containing solution in 1\% BSA in PBS with $2 \mathrm{mM}$ sodium azide overnight at $4^{\circ} \mathrm{C}$. After rinsing in $\mathrm{PBS}$ for 5 min $\times 3$, samples were stained with secondary antibody and Hoescht 33342 for $2 \mathrm{~h}$.

10 For Trio staining, the samples were stained according to the Alexa tyramide amplification system with the SuperBoost protocol (Thermo Fisher Scientific). Samples were then rinsed in PBS for 5 min $\times 2$ /Milli-Q water 5 min $\times 1$ and mounted on Vectashield Vibrance (Vector Laboratories) or Prolong Fade Glass (Thermo Fisher Scientific).

For mouse brain samples, mice were deeply anesthetized with isoflurane inhalation or

15 ketamine/xylazine (200 mg/kg ketamine and $20 \mathrm{mg} / \mathrm{kg}$ xylazine) intra-peritoneal injection and intracardially perfused with 4\% PFA in phosphate-buffered saline (PBS). The brains were removed and post-fixed in $4 \%$ PFA in PBS overnight at $4^{\circ} \mathrm{C}$. Samples were then rinsed in PBS x3, 15 min each. Serial sections of $50 \mu \mathrm{m}$ or $200 \mu \mathrm{m}$ were obtained on a vibratome. For $50 \mu \mathrm{m}$ mouse brain sections, fixed slices were incubated at room

20 temperature in $0.3 \%$ Triton-X in PBS overnight, followed by $5 \mathrm{~h}$ in blocking buffer (BlockAid, Thermo Fisher Scientific), overnight in primary antibody, and again overnight in secondary antibody and Hoechst $33342(2 \mu \mathrm{g} / \mathrm{ml})$. Both primary and secondary antibodies were diluted in the same blocking buffer (BlockAid, Thermo Fisher Scientific). The stained sections were then rinsed in PBS $15 \times 2$ times/300 mOsm glycerol $15 \mathrm{~min} x$

25 1, and mounted in Prolong Fade Glass (Thermo Fisher Scientific). Between antibodies, slices were washed x3, 15 min each with PBS. For $200 \mu \mathrm{m}$ mouse brain sections, slices were first treated with the epoxide-crosslinker as in the SHIELD protocol (Life Canvas Technology, Park et al, 2018). Afterwards, sections were incubated in $2 \%$ Triton-X in PBS with $2 \mathrm{mM}$ sodium azide for 3 days for permeabilization. Sections were then incubated in

30 primary and secondary antibodies for 2 days at room temperature, respectively. After washing, sections were mounted in SlowFade Glass (Thermo Fisher Scientific).

The following primary antibodies were used: rabbit anti-GFP (Chromotek PABG1, 1:1000), guinea-pig anti-SERT (synaptic systems 340004, 1:200), mouse anti-ADCY3 (Encor Biotechnology MCA-1A12, 1:1000), rabbit anti-PCP4 (Millipore Sigma, HPA005792,

35 1:500), chicken anti-rootletin (Millipore Sigma, ABN1686, 1:1000), rabbit anti-ADD1 (Abcam EP734Y, \#ab40760, 1:250), rabbit anti-synaptophysin (Cell Signaling, \#36406, 1:100), rabbit anti-synaptophysin (Thermo Fisher Scientific, MA5-14532, 1:200), rabbit anti-Trio GEFD2 (custom antibody, gift from Dr. Susanne Schmidt, 1:500). The following secondary antibodies and dyes were used: Alexa Fluor Plus 488 goat anti-rabbit (1:1000,

40 Thermo Fisher Scientific), Alexa Fluor 488 donkey anti-rabbit (1:400, highly-cross absorbed, Jackson ImmunoResearch), CF488 donkey anti-guinea pig (1:1000, Biotium), 
Alexa Fluor Plus 555 goat anti-rabbit (1:1000, Thermo Fisher Scientific), CF633 goat antimouse $\operatorname{lgG}_{1}$ (1:1000, Biotium), Hoescht $33342(2 \mu \mathrm{g} / \mathrm{ml}$, Thermo Fisher Scientific), SuperBoost goat anti-rabbit polyHRP (ready-to-use $1 \mathrm{x}$ concentration, Thermo Fisher Scientific).

5 All confocal imaging employed a Zeiss 880 Laser Scanning Confocal Microscope (LSM) equipped with a Plan-Apochromat $63 x$ oil objective (Zeiss, NA = 1.40), 40x multiimmersion LD LCI Plan-Apochromat objective (Zeiss, NA = 1.20), or 20x air PlanApochromat objective (Zeiss, NA = 0.8); ZEN Black software (Zeiss). Hoechst 3342 was excited by $405 \mathrm{~nm}$ laser light and the spectral detector set to 409-481 $\mathrm{nm}$. Alexa $488 / C F 488$ was excited by $488 \mathrm{~nm}$ laser light and the spectral detector set to 490-545 $\mathrm{nm}$. Alexa 555 was excited by $561 \mathrm{~nm}$ laser light and the spectral detector set to 570-642 $\mathrm{nm}$. ATTO-590 was excited by $594 \mathrm{~nm}$ laser light (Airyscan only). CF633 was excited with $633 \mathrm{~nm}$ light and the spectral detector set to $642-755 \mathrm{~nm}$. The spectral detector was only used for non-Airyscan confocal scanning imaging sessions.

15 Sample preparation, FIB-SEM imaging, and data analyses of mouse brain samples

\section{Conventional chemical fixation protocol}

Mice were anesthetized with ketamine/xylazine $(200 \mathrm{mg} / \mathrm{kg}$ ketamine and $20 \mathrm{mg} / \mathrm{kg}$ xylazine) intra-peritoneal injection and perfused with a solution of $2 \%$ PFA and $2 \%$ 20 glutaraldehyde in $0.1 \mathrm{M}$ sodium cacodylate buffer, $0.2 \mathrm{mM} \mathrm{CaCl}_{2}$. The brain samples were dissected and post-fixed in the perfusion solution overnight at $4^{\circ} \mathrm{C}$. After rinsing with $0.1 \mathrm{M}$ sodium cacodylate buffer, $300 \mu \mathrm{m}$ serial sections were obtained on a vibratome. The tissue was then immersed in $1 \%$ osmium tetroxide and $1.5 \%$ potassium ferricyanide in a $0.1 \mathrm{M}$ cacodylate buffer for $1 \mathrm{~h}$. After rinsing in $0.1 \mathrm{M}$ sodium cacodylate buffer, the 25 sections were further stained with $1 \%$ osmium tetroxide in water for $1 \mathrm{~h}$, followed by $2 \%$ uranyl acetate in maleate buffer $(\mathrm{pH}=5.15)$ overnight at $4^{\circ} \mathrm{C}$. The tissue was then washed in water, dehydrated with graded ethanol, and embedded in Epon812 resin.

Epon-812 flat-embedded mouse hippocampal CA1 samples were first mounted on an aluminum stub. The sample surface was polished on an ultramicrotome, followed by carbon coating $(20 \mathrm{~nm})$. The samples were then imaged on the Zeiss Crossbeam 540 at 5-6 nm pixel size with 15-20 nm milling using ATLAS 5 software (Zeiss).

\section{Hybrid protocol}

2- to 3-month-old male C57/BL6 mice were deeply anesthetized and transcardially perfused with $30 \mathrm{~mL}$ of $3 \%$ PFA $(60 \mathrm{mM} \mathrm{NaCl}, 130 \mathrm{mM}$ glycerol, $10 \mathrm{mM}$ sodium phosphate buffer). The brain was carefully dissected from the skull and post-fixed with 50 $\mathrm{mL}$ of $3 \%$ PFA (30 mM NaCl, $70 \mathrm{mM}$ glycerol, $30 \mathrm{mM}$ PIPES buffer, $10 \mathrm{mM}$ betaine, 2 $\mathrm{mM} \mathrm{CaCl}_{2}, 2 \mathrm{mM} \mathrm{MgSO}_{4}$ ) at room temperature for $2 \mathrm{~h}$. The brain sample was then rinsed in a 400 mOsM buffer $(65 \mathrm{mM} \mathrm{NaCl}, 100 \mathrm{mM}$ glycerol, $30 \mathrm{mM}$ PIPES buffer, $10 \mathrm{mM}$ betaine, $2 \mathrm{mM} \mathrm{CaCl}_{2}$, and $2 \mathrm{mM} \mathrm{MgSO}_{4}$ ) for $0.5 \mathrm{~h}$, followed by vibratome sectioning 
(coronal sections, $100 \mu \mathrm{m}$ thickness) using a Leica VT1000S vibratome in the same buffer. $100 \mu \mathrm{m}$ sections were then fixed in 1\% PFA, $2 \%$ glutaraldehyde solution $(30 \mathrm{mM} \mathrm{NaCl}$, $70 \mathrm{mM}$ glycerol, $30 \mathrm{mM}$ PIPES buffer, $10 \mathrm{mM}$ betaine, $2 \mathrm{mM} \mathrm{CaCl}$, $2 \mathrm{mM} \mathrm{MgSO}_{4}, 75$ $\mathrm{mM}$ sucrose) overnight at $4^{\circ} \mathrm{C}$. Sections were then washed using the $400 \mathrm{mOsM}$ rinsing buffer (see above). Round samples of the hippocampus were created from the $100 \mu \mathrm{m}$ coronal sections using a $2 \mathrm{~mm}$ biopsy punch (Miltex). The $2 \mathrm{~mm}$ samples were dipped in 1-Hexadecene, placed in a $100 \mu \mathrm{m}$ aluminum carrier, covered with a flat carrier and highpressure frozen using a Wohlwend compact high-pressure freezer (Wohlwend $\mathrm{GmbH}$, Switzerland). Samples were then freeze-substituted in $0.5 \%$ osmium tetroxide, $20 \mathrm{mM} 3-$ amino-1,2,4-triazole or $20 \mathrm{mM}$ imidazole, $0.1 \%$ uranyl acetate, $4 \%$ water in acetone, using a Leica AFS2 system. Specimens were further dehydrated in $100 \%$ acetone and embedded in Durcupan resin.

Two datasets were acquired using the hybrid protocol, stained with either osmiumimidazole or osmium-3-amino-1,2,4-triazole. The samples were mounted on a copper post and trimmed to the Region of Interest (ROI), guided by X-ray tomography data obtained by a Zeiss Versa XRM-510. The samples were coated with a thin layer of 10- to 20-nm gold and 50- to 100-nm carbon and imaged by a customized Zeiss Merlin FIBSEM or NVision40 FIB-SEM system using $8 \mathrm{~nm}$ pixel size with 2 or $4 \mathrm{~nm}$ of milling depth. After alignment using a Scale Invariant Feature Transform (SIFT) based algorithm (Lowe, 2004), the stacks were binned by a factor of 2 or 4 along $z$ to form a final isotropic volume of $35 \mu \mathrm{m} \times 35 \mu \mathrm{m} \times 40 \mu \mathrm{m}$ and $50 \mu \mathrm{m} \times 50 \mu \mathrm{m} \times 44 \mu \mathrm{m}$ with $8 \mathrm{~nm} \times 8 \mathrm{~nm} \times 8 \mathrm{~nm}$ voxels.

The electron microscopy datasets generated above were manually segmented using VAST (Volume Annotation and Segmentation Tool, Berger et al., 2018). Segmented results were exported as obj files, and rendered using 3ds Max 2021 (Autodesk, Inc.).

25 For synaptic vesicles in Figure 2E, the centroids of segmented vesicles were calculated in 3D, and $40 \mathrm{~nm}$ spheres were generated as $40 \mathrm{~nm}$ spheres in $3 \mathrm{ds}$ Max.

The hybrid protocol was developed for this work, which were used to generate two enhanced FIB-SEM datasets in the CA1 area to examine primary cilia. Prior to the completion of this manuscript, these datasets have been shared with others to examine

30 lipid droplets (loannou et al., 2019), myeline distribution along large axons (Gao et al., 2019), and mitochondrial morphology (Thomas et al., 2019). All FIB-SEM datasets will be available upon request.

\section{Characterization of GRAB-HTR6-PM sensor}

\section{Expression of the GRAB-HTR6-PM sensor in HEK293T cells}

35 HEK293T cells were cultured in DMEM (Gibco) supplemented with 10\% (v/v) FBS (Gibco) and $1 \%$ penicillin-streptomycin (Gibco) at $37^{\circ} \mathrm{C}$ in $5 \% \mathrm{CO}_{2}$. HEK293T cells were plated on 96-well plates and transfected with a mixture of plasmids and PEI (300 ng plasmids and 900 ng PEI for each well) when the cells were grown to $\sim 70 \%$ confluence. The medium was replaced after 4-6 h, and cells were used for imaging $24 \mathrm{~h}$ after transfection. 
Fluorescence imaging of HEK293T cells expressing the GRAB-HTR6-PM sensor

The DNA for the IRES-mCherry-CAAX cascade was fused downstream of the GRABHTR6-PM sensor to normalize the expression level of the sensor and calibrate the membrane signal. HEK293T cells expressing the GRAB-HTR6-PM sensor were imaged by the Opera Phenix high-content screening system. Before imaging, the culture medium was replaced with $100 \mu$ l Tyrode's solution. For imaging, a 40x, 1.1-NA water-immersion objective, a 488-nm laser combined with a 525/50-nm emission filter for excitation and collection of the GFP fluorescence signal, and a 561-nm laser combined with a 600/30$\mathrm{nm}$ emission filter for the collection of the mCherry fluorescence signal were used. The same field of views (FOVs) were imaged without or with the application of 5-HT (at various concentrations, in Tyrode's solution), respectively. The fluorescence signal of the GRABHTR6-PM sensor was calibrated using the ratio of GFP to mCherry.

\section{Quantification and statistical analysis of GRAB-HTR6-PM sensor}

Images collected by an Opera Phenix high-content screening system from cultured

15 HEK293T cells were processed using ImageJ (1.53c) software (NIH) and analyzed using custom-written MATLAB (R2020b) codes. The fluorescence response $\left(\Delta F / F_{0}\right)$ was calculated using the formula $\left(F-F_{0}\right) / F_{0}$, in which $F_{0}$ is the baseline fluorescence signal after subtracting the background. The dose-response curve was plotted using OriginPro (2020b).

\section{Measurements of the GRAB-HTR6-cilia sensor}

\section{GRAB-HTR6-cilia titration curve}

Serum deprived, JF552 labeled RPE-1 cells stably expressing GRAB-HTR6-cilia were imaged in a HEPES-buffered imaging media $(140 \mathrm{mM} \mathrm{NaCl}, 20 \mathrm{mM} \mathrm{HEPES,} 2.5 \mathrm{mM} \mathrm{KCl}$, $1.8 \mathrm{mM} \mathrm{CaCl}_{2}, 1.0 \mathrm{mM} \mathrm{MgCl}$, $\mathrm{pH}=7.4, \mathrm{mOsm}=300$; Live Cell Imaging Solution, Thermo

25 Fisher Scientific A14291DJ) using a 20x air Plan-Apochromat objective (Zeiss, NA = 0.8) with FAST Airyscan on a Zeiss 880 confocal microscope at $37^{\circ} \mathrm{C}$. GFP and JF552 were excited with $488 \mathrm{~nm}$ and $561 \mathrm{~nm}$ lasers, respectively. Keeping the same field of view, $Z$ stacks were acquired at different concentrations of 5-HT diluted in the same imaging buffer. The two channels were aligned using Zen Blue (Zeiss). Cilia were segmented using CiliaQ based on the JF552 channel (Hansen et al., 2021). Per cilium GFP mean fluorescent intensities were then calculated for different concentrations. The fluorescence response $\left(\Delta F / F_{0}\right)$ was calculated using the formula $\left(F-F_{0}\right) / F_{0}$, in which $F_{0}$ is the baseline fluorescence signal after subtracting the background. The dose-response curve was plotted using Graphpad Prism 9.2.

\section{GRAB-HTR6-cilia in hippocampal-midbrain neuron co-culture}

Hippocampal neurons expressing GRAB-HTR6-cilia and serotonergic neurons expressing ChrimsonR-tdTomato, farnesylated SNAP-Tag:JF552, or tdTomato were imaged with a 40x multi-immersion LD LCI Plan-Apochromat objective (Zeiss, NA = 1.2; silicone oil was used as the immersion media) on a Zeiss LSM 880 microscope in artificial 
cerebral spinal fluid ( $\mathrm{NaCl} 124 \mathrm{mM}, \mathrm{KCl} 2.5 \mathrm{mM}, \mathrm{NaH}_{2} \mathrm{PO}_{4} 1.2 \mathrm{mM}, \mathrm{NaHCO}_{3} 24 \mathrm{mM}$, HEPES $5 \mathrm{mM}$, glucose $12.5 \mathrm{mM}, \mathrm{MgSO}_{4} 2 \mathrm{mM}, \mathrm{CaCl}_{2} 2 \mathrm{mM}$, Ting et al., 2018) at $37^{\circ} \mathrm{C}$. Two-channel FAST Airyscan images were first imaged to characterize the axociliary synapses using $488 \mathrm{~nm}$ and 561 excitations for GFP and red fluorophores, respectively. GRAB-HTR6-cilia was then imaged at $1 \mathrm{~Hz}$ using the $488 \mathrm{~nm}$ laser with Z-stacks. After $30 \mathrm{~s}$ (frame = 31), the $594 \mathrm{~nm}$ laser line was used to photostimulate at the same $1 \mathrm{~Hz}$ frequency (repetition $=1$, same pixel dwell time as imaging) as image acquisition (488 $\mathrm{nm}$ ). A total of 120 Z-stacks were acquired per cilium (120 s). Airyscan stacks were processed using Zen Black (auto-strength, 3D; Zeiss). These 4D stacks (XYZT) were projected onto single planes (XYT) by maximum intensity projection in Fiji/lmageJ. Cilia were segmented using CiliaQ (Hansen et al, 2021). The first 10 time points, which often showed significant quenching, were discarded for subsequent analyses. A 1- $\mu \mathrm{m}$ circle at the contact site (ChrimsonR, tdTomato, or SNAP:JF522) or at the site at which the cilium is closest to a serotonergic axon was used as the region of interest (ROI) to calculate

15 mean intensity values over time (ImageJ/Fiji). These values were processed with a low pass filter at $0.2 \mathrm{~Hz}$. The average intensity of the 10 time points prior to photostimulation was used as the baseline fluorescence intensity, or $F_{0}$. The maximum $\Delta F / F_{0}$ during photostimulation was used for statistical comparisons in different conditions using estimation statistics (Ho et al., 2019). Low pass filter, baseline fluorescence intensity calculation, and estimation statistics were done in a custom Python code.

\section{HTR6-RhoA FRET measurements and analysis}

Images were collected using silicone-oil immersion media with a 40x multi-immersion LD LCI Plan-Apochromat objective (Zeiss, NA = 1.2; silicone oil immersion media) on a Zeiss LSM 880 microscope. Single apical cilia were imaged at zoom 10 with 4 Airy units (146 $\mu \mathrm{m}$ pinhole), $212 \times 212$ frame size, $0.1 \mu \mathrm{m}$ pixel size, $0.93 \mu$ s pixel dwell time, 16-bit bidirectional scanning, and 4 optical sections $(1.8 \mu \mathrm{m})$ to cover the entire length of the cilium. The resulting temporal resolution was $0.25 \mathrm{~s}$. The donor fluorophore, sGFP2 was excited by the $488 \mathrm{~nm}$ laser. Donor emission from mScarlet-I was collected with the spectral detector set to $490-550 \mathrm{~nm}$. Acceptor-sensitized emission was collected with the spectral detector set to $570-650 \mathrm{~nm}$.

For uncaging experiments, the following uncaging parameters were used on a $5 \mu \mathrm{m}$ radius circle adjacent to the ciliary tip: repeat each stack $\times 10$ using the same pixel dwell time as during scanning. Two experiment blocks (400 timeframes each) were used to acquire data with and without uncaging. Uncaging started at frame 20 in the first block, with no uncaging during the second block.

The 4D stacks (XYZT) were first projected onto single planes (XYT) by maximum intensity projection in Fiji/lmageJ. Datasets with bidirectional scanning artefacts (i.e. misalignment between two scanning directions in alternating lines), significant movements, and/or focus drifts were excluded. The donor channel was used to create a mask to segment the cilia

40 ("cilia mask") by using the "subtract background with smoothing paraboloid" command followed by Otsu thresholding in Fiji/lmageJ. The donor and sensitized emission intensity 
values were individually subtracted by using the mean intensity from an acellular area outside the cilium. These background-subtracted images were then masked using the cilia mask to isolate/segment cilia-specific signals. The FRET ratio was calculated by dividing the segmented sensitized emission by segmented donor emission. For direct ligand application experiments, FRET recordings were digitized at $4 \mathrm{~Hz}$ and filtered at 1 $\mathrm{Hz}$ with a low pass Fourier transform digital filter implemented in Origin (OriginLab Corporation). For uncaging experiments, the FRET data was processed with temporal averaging by a factor of 2 . Change of the mean FRET ratio of the entire cilium was plotted using the $\mathrm{Z}$ plot function in Fiji/lmageJ and imported into Excel spreadsheets. The

10 changes were visualized using PlotTwist (Goedhart, 2020). $\Delta F / F$ was calculated by using the mean FRET ratio at the beginning of the experiments as the baseline (frame 1 to 25 for direct ligand application, and 1 to 30 for uncaging experiments). Statistical testing of RhoA spikes were done in Prism 8 (GraphPad).

\section{Ciliary-RhoA FLIM measurements and analysis}

15 3D Z stacks were collected using an 40x oil immersion Plan-Apochromat objective (Leica, $N A=1.3$ ) on a Leica SP8 Falcon microscope at $2 x$ the Nyquist limit and 1 Airy disc. Donor sGFP fluorescence was excited by a pulsed $(40 \mathrm{MHz})$ white light laser tuned at $488 \mathrm{~nm}$, and emitted photons between $490 \mathrm{~nm}$ and $550 \mathrm{~nm}$ were collected with line repetition $=8$. To calculate the fluorescence lifetime of unquenched and quenched donor, large

20 populations of cells expressing the RhoA sensor were imaged, and fluorescence lifetimes were calculated by the n-exponential reconvolution fitting algorithm (2 exponential components) with pixel binning by a factor of 2 (Leica LAS X FLIM/FCS software v3.5). The fluorescence lifetime of quenched and unquenched donor was determined to be 1.3 and $2.7 \mathrm{~ns}$, respectively. These numbers were then used for all subsequent fittings. Each

25 cilium is imaged individually with Z-stacks. To calculate representative RhoA sensor sGFP fluorescence lifetime per cilium, "FLIM Image Fit" was performed in the Leica LAS $X$ FLIM/FCS software suite. The resulting datasets were rendered in 3D for visualization (Figure 5A, B, D, F, G, I, and J) and exported as two-channel stacks, encoding photon counts and fluorescence lifetime, respectively. Subsequent imaging analyses were done

30 in Python. For each stack, the channel encoding counts were used to segment cilia (Otsu and Yen thresholding were used for HEK cells and neurons, respectively). The segmented cilia were then used as masks to extract ciliary voxels from the FLIM channel. Voxels with less than 50 counts were discarded. An alpha distribution was fitted to the histogram of the FLIM channel, as it provided the best fitting among all 80 different

35 statistical distributions tested. The peak of the alpha distribution was used as the mode, or the representative FLIM of a given cilium. Statistical comparisons of cilia from different cell types and states, and after stimulation were performed using estimation statistics (Ho et al., 2019).

\section{D ATAC-Airy staining and analysis}

40 Transposase-mixture solution (hyperactive Tn5 transposase with ATTO-590 or ATT-565 conjugated oligos) were prepared as described previously (Chen et al., 2016). $50 \mu \mathrm{m}$ 
thick, PFA-fixed CA1 coronal sections from 6-month-old C57BL/6 wild-type, HTR6 KO, non-doxycycline-treated control and doxycycline-treated HTR6-TRIP mice were obtained using the same method as described above. A $3 \mathrm{~mm}$ punch of the CA1 area was created using a biopsy punch on the $50 \mu \mathrm{m}$ thick sections (Electron Microscopy Sciences). These punches were permeabilized in $0.3 \%$ Triton/PBS overnight at RT and blocked with buffer (BlockAid, Thermo Fisher Scientific) for $1 \mathrm{~h}$ at RT. The samples were then rinsed in PBS for $15 \mathrm{~min} \times 3$ and incubated in the transposase-mixture solution $(100 \mathrm{nM}, 10 \mathrm{mM}$ Tris$\mathrm{HCL} \mathrm{pH} \mathrm{7.5,} 10 \mathrm{mM} \mathrm{MgCl}_{2}, 25 \%$ dimethylformamide) overnight at $4^{\circ} \mathrm{C}$, followed by $1 \mathrm{~h}$ at $37^{\circ} \mathrm{C}$ on a rotator. After incubation, the samples were washed $\times 3$ for 15 min at $55^{\circ} \mathrm{C}$ with

$101 \times$ PBS containing $0.01 \%$ SDS and $50 \mathrm{mM}$ EDTA, followed by regular PBS at room temperature for $15 \mathrm{~min}$. The samples were subsequently stained with Hoechst 33342 (2 $\mathrm{ug} / \mathrm{ml}$ ) for $2 \mathrm{~h}$, rinsed in PBS $15 \times 2 / 300$ mOsm glycerol x1, and mounted in Prolong Fade Glass (Thermo Fisher Scientific). Non-doxycycline-treated control and doxycyclinetreated HTR6-TRIP samples were also stained with ADCY3 and CF633 (see above).

15 For quantification of ATAC-see intensity per nucleus, CA1 areas were imaged using a 40x multi-immersion LD LCI Plan-Apochromat objective (Zeiss, NA = 1.2; doxycyclinetreated control and doxycycline-treated HTR6-TRIP) or a 63x Plan-Apochromat oilimmersion objective (WT and KO) using FAST Airyscan or Airyscan, respectively. The FAST Airyscan and Airyscan stacks were processed with 3.4 and 6.7 filter strength/3D mode in Zen Black or Zen Blue, respectively (Zeiss; voxel size $41 \mathrm{~nm} \times 41 \mathrm{~nm} \times 1939 \mathrm{~nm}$ and $35 \mathrm{~nm} \times 35 \mathrm{~nm} \times 144 \mathrm{~nm}$ ). The stacks were then downsampled to a voxel size of 400 $\mathrm{nm} \times 400 \mathrm{~nm} \times 400 \mathrm{~nm}$ and imported into Cellpose (Stringer et al., 2021). The Hoechst channel was used to segment each individual nucleus using "cyto2". Segmentations less than 5000 voxels (equivalent to a sphere with an $8-\mu \mathrm{m}$ diameter) were excluded, and the mean intensities of the nuclei were calculated in a custom Python code for statistical analyses by estimation statistics (Ho et al, 2019).

\section{Single Cell ATAC-seq}

One frozen hippocampus each from KO and wild-type mice were prepared for scATACseq according to the manufacturer's protocol for flash frozen mouse brain (Protocol 2.2,

30 CG000212 Rev B, 10X Genomics). Nuclei were counted using a Luna-II automated cell counter (Logos Biosystems). Approximately 12,000 nuclei per sample were subjected to the Chromium NextGem scATAC-seq v1.1 assay (10X Genomics) and the resulting libraries were sequenced on a NextSeq 550 (Illumina) with 50 bp read 1, 8 bp i7 index read, $16 \mathrm{bp}$ i5 index read, and 50 bp read 2 . The KO library was sequenced a second time to increase read depth, and both runs were combined during analysis.

Sample demultiplexing and initial scATAC-seq analysis including read alignment and peak calling were performed using cellranger-atac mkfastq and cellranger-atac count, respectively, using v1.2.0 software and the associated mm10 genome reference ('refdatacellranger-atac-mm10-1.2.0'; 10X Genomics). Count data for each sample were imported 40 into Seurat v4.0.4 (Butler et al., 2018) in $\mathrm{R}$ version 4.1.1 using cell barcodes discovered by the cellranger-atac pipeline. The two datasets were merged, normalized, and clustered 
using Signac v1.3.0 (Stuart et al., 2020). Differential peak accessibility and gene activity scores (accessibility within gene bodies and promoter areas) were calculated for each initial cluster and were used to assign cell identities. Clusters were then merged by cell identity. Differential peaks and gene activities were re-calculated for the merged clusters. GREAT analysis was used to calculate distances of the peaks to transcription starting sites and extract affected nearby genes (McLean et al, 2010). Gene ontology and Reactome analyses were performed using g:Profiler (Raudvere et al, 2019).

\section{QUANTIFICATION AND STATISTICAL ANALYSIS}

Quantification and statistical analyses of GRAB-HTR6, ciliary RhoA FRET/FLIM, ATACAiry, and scATAC-seq were described above in their respective sections. Details of experiments, including sample size and statistics can be found in the text, figures, or figure legends. Except for Figure 3G-H, all statistical tests were done using estimation statistics (Ho et al, 2019) or permutation test comparing different distributions (Figure $3 \mathrm{H})$. In most cases, conventional p-values were also provided. No method was used to predetermine sample size $(\mathrm{N})$, which represents data collected spanning different sessions. Blinding was not performed. Formal randomization techniques were not used.

\section{Quantification of cilia trajectory in the brain}

3D stacks of ADCY3-stained cilia images were projected along the $z$ axis using maximum intensity projections and analyzed with the OrientationJ plug-in in ImageJ/Fiji using a

20 gaussian gradient (Püspöki et al., 2016; Rezakhaniha et al., 2012). The algorithm computes the structure tensor for each pixel in the image using a sliding gaussian analysis window. A local window size of 2 and 20 pixels are used for visualization and weighted histogram calculation, respectively. Gaussian fitting of the weighted histogram was performed and plotted in Graphpad Prism (v9.2).

25 Quantification of cilia and serotonergic axon, synaptophysin vesicle apposition and nuclear adducin puncta

\section{Linear Unmixing}

3D stacks of Hoechst-stained nuclei, SERT-stained serotonergic axons (CF488), synaptophysin-stained presynaptic terminals (Alexa Fluor Plus 555), and ADCY3-stained cilia (CF633) images were acquired using FAST Airyscan. The red and far-red channel Airyscan data $(41 \mathrm{~nm} \times 41 \mathrm{~nm} \times 189 \mathrm{~nm}$ voxel size, 3D Airyscan processing with Auto Filter) were unmixed via a custom MATLAB script.

Nucleus, cilia, axon and synaptophysin segmentation

The multichannel volumes acquired were resampled to generated isotropic voxel sizes of $35189 \mathrm{~nm}$. The nucleus, cilia, and axon signals were segmented using Otsu's methods to threshold, followed by dilation and erosion operations to join fragmented structures, and finally filtered to remove small discrete objects with no biological relevance. Nuclei that were not part of the pyramidal neuron cell layer were removed by dilating the dense collection of nuclei, and subsequently retaining just the largest connected object 
(corresponding to pyramidal neuron cell layer). Only nuclei and associated cilia with the pyramidal neuron cell layer were included for downstream analysis steps. The central axes of segmented cilia and axon masks were computed (skeletonized), which also served to separate and identify distinct cilia. The cilia near the imaged volume boundaries or those which were associated with non-pyramidal neuron cell layer nuclei were excluded from further analyses. The synaptophysin and ADD1 puncta positions were determined by detecting their local signal maxima using a 3D Laplacian-of-Gaussian filter described previously (Aguet et al., 2016). Nucleus centers were determined by eroding the segmented nuclei masks, followed by calculating the distance transformation and

10 thresholded to separate touching nuclei. The centroids of these discrete eroded nuclei were used to define the centers of search for ADD1 puncta with varying radii between 1 $-8 \mu \mathrm{m}$.

\section{Calculation of axon-cilia and SERT+ synaptophysin -cilia distance}

3D distance transformations of the nuclei, skeletonized axon, and synaptophysin masks were calculated and multiplied by the skeletonized cilia masks. The resulting mask encoded the distance information for the respective transformation mask in the medial axes of the cilia. As serotonergic varicosities are between 1 and $3 \mu \mathrm{m}$ (Alvarez et al., 1998), we used $2 \mu \mathrm{m}$ between skeletonized cilia and skeletonized axons as the cutoff for axon-contacting and non-axon-contacting cilia. Subsequently, synaptophysin puncta within $1 \mu \mathrm{m}$ of skeletonized serotonergic axon central axes are considered associated with serotonergic axons (SERT-positive). For the visualization of the distance-encoded cilia, the cilia central axes were dilated using a sphere morphological structural kernel with a radius of 1 pixel. The resulting volumes were then projected to only visualize the distance information using a custom lookup table (Figure 3C-D). Furthermore, the total cilia length and the corresponding distance to the closest axon was plotted as a 2D density scatter using MATLAB (Chung, 2021) with a marker size set to 10 and a "jet" colormap (Figure 3F-G). Violin plots of cilium length for contacting and non-contacting cilia (Figure 3H) were generated using PlotsOfData (Postma and Goedhart, 2019). Statistical comparison of the two groups were done in GraphPad Prism. Permutation tests to compare these two distributions were performed using Mlxtend in Python (Raschka, 2018). 


\section{References}

Aguet, F., Upadhyayula, S., Gaudin, R., Chou, Y., Cocucci, E., He, K., Chen, B.-C., Mosaliganti, K., Pasham, M., Skillern, W., et al. (2016). Membrane dynamics of dividing cells imaged by lattice light-sheet microscopy. Mol Biol Cell 27, 3418-3435.

5 Alvarez, F.J., Pearson, J.C., Harrington, D., Dewey, D., Torbeck, L., and Fyffe, R.E.W. (1998). Distribution of 5-hydroxytryptamine-immunoreactive boutons on a-motoneurons in the lumbar spinal cord of adult cats. J Comp Neurol 393, 69-83.

Arellano, J.I., Guadiana, S.M., Breunig, J.J., Rakic, P., and Sarkisian, M.R. (2012). Development and distribution of neuronal cilia in mouse neocortex. Journal of Comparative Neurology 520, 848-873.

Armbruster, B.N., Li, X., Pausch, M.H., Herlitze, S., and Roth, B.L. (2007). Evolving the lock to fit the key to create a family of $G$ protein-coupled receptors potently activated by an inert ligand. Proc National Acad Sci 104, 5163-5168.

Ashburner, M., Ball, C.A., Blake, J.A., Botstein, D., Butler, H., Cherry, J.M., Davis, A.P., Dolinski, K., Dwight, S.S., Eppig, J.T., et al. (2000). Gene Ontology: tool for the unification of biology. Nat Genet 25, 25-29.

Baldi, P., Alhassen, W., Chen, S., Nguyen, H., Khoudari, M., and Alachkar, A. (2021). Large-scale analysis reveals spatiotemporal circadian patterns of cilia transcriptomes in the primate brain. J Neurosci Res Advance online publication.

20 Bandyopadhyay, U., Cotney, J., Nagy, M., Oh, S., Leng, J., Mahajan, M., Mane, S., Fenton, W.A., Noonan, J.P., and Horwich, A.L. (2013). RNA-Seq profiling of spinal cord motor neurons from a presymptomatic SOD1 ALS mouse. Plos One 8, e53575.

Barnett, S., Reilly, S., Carr, L., Ojo, I., Beales, P., and Charman, T. (2002). Behavioural phenotype of Bardet-Biedl syndrome. J Med Genet 39, e76.

25 Barreiro-Iglesias, A., Mysiak, K.S., Scott, A.L., Reimer, M.M., Yang, Y., Becker, C.G., and Becker, T. (2015). Serotonin promotes development and regeneration of spinal motor neurons in zebrafish. Cell Reports 13, 924-932.

Belmer, A., Klenowski, P.M., Patkar, O.L., and Bartlett, S.E. (2017). Mapping the connectivity of serotonin transporter immunoreactive axons to excitatory and inhibitory

30 neurochemical synapses in the mouse limbic brain. Brain Structure and Function 222, $1297-1314$.

Bennett, V., and Gilligan, D.M. (1993). The spectrin-based membrane skeleton and micron-scale organization of the plasma membrane. Annu Rev Cell Biol 9, 27-66.

Berger, D.R., Seung, S.H., and Lichtman, J.W. (2018). VAST (Volume Annotation and Segmentation Tool): efficient manual and semi-automatic labeling of large 3D image stacks. Front Neural Circuit 12, 88. 
Bindels, D.S., Haarbosch, L., Weeren, L. van, Postma, M., Wiese, K.E., Mastop, M., Aumonier, S., Gotthard, G., Royant, A., Hink, M.A., et al. (2017). mScarlet: a bright monomeric red fluorescent protein for cellular imaging. Nature Methods 14, 53-56.

Bishop, G.A., Berbari, N.F., Lewis, J., and Mykytyn, K. (2007). Type III adenylyl cyclase localizes to primary cilia throughout the adult mouse brain. J Comp Neurology 505, 562-571.

Boess, F.G., Monsma, F.J., Carolo, C., Meyer, V., Rudler, A., Zwingelstein, C., and Sleight, A.J. (1997). Functional and radioligand binding characterization of rat 5-HT6 receptors stably expressed in HEK293 cells. Neuropharmacology 36, 713-720.

10 Bornens, M. (2012). The Centrosome in Cells and Organisms. Science 335, 422-426.

Bouquier, N., Fromont, S., Zeeh, J.-C., Auziol, C., Larrousse, P., Robert, B., Zeghouf, M., Cherfils, J., Debant, A., and Schmidt, S. (2009). Aptamer-derived peptides as potent inhibitors of the oncogenic RhoGEF Tgat. Chem Biol 16, 391-400.

Breitinger, H.-G.A., Wieboldt, R., Ramesh, D., Carpenter, B.K., and Hess, G.P. (2000).

15 Synthesis and characterization of photolabile derivatives of serotonin for chemical kinetic investigations of the serotonin 5- $\mathrm{HT}_{3}$ receptor. Biochemistry 39, 5500-5508.

Brodsky, M., and Neumaier, J.F. (2017). 5-HT6 receptor blockade regulates primary cilia morphology in striatal neurons. Brain Research 1660, 10-19.

Butler, A., Hoffman, P., Smibert, P., Papalexi, E., and Satija, R. (2018). Integrating

20 single-cell transcriptomic data across different conditions, technologies, and species. Nat Biotechnol 36, 411-420.

Chen, C.-L., Lin, Y.-P., Lai, Y.-C., and Chen, H.-C. (2011). a-adducin translocates to the nucleus upon loss of cell-cell adhesions. Traffic 12, 1327-1340.

Chen, X., Shen, Y., Draper, W., Buenrostro, J.D., Litzenburger, U., Cho, S.W.,

25 Satpathy, A.T., Carter, A.C., Ghosh, R.P., East-Seletsky, A., et al. (2016). ATAC-see reveals the accessible genome by transposase-mediated imaging and sequencing. Nat Methods 13, 1013-1020.

Chung, M. (2021). Density scatter plot - File Exchange - MATLAB Central.

Conduit, S.E., and Vanhaesebroeck, B. (2020). Phosphoinositide lipids in primary cilia 30 biology. Biochem J 477, 3541-3565.

The Gene Ontology Consortium, Carbon, S., Douglass, E., Good, B.M., Unni, D.R., Harris, N.L., Mungall, C.J., Basu, S., Chisholm, R.L., Dodson, R.J., et al. (2020). The Gene Ontology resource: enriching a GOld mine. Nucleic Acids Res 49, D325-D334.

Delling, M., DeCaen, P.G., Doerner, J.F., Febvay, S., and Clapham, D.E. (2013). 
Deo, C., Sheu, S.-H., Seo, J., Clapham, D.E., and Lavis, L.D. (2019). Isomeric tuning yields bright and targetable red Ca2+ indicators. J Am Chem Soc 141, 13734-13738.

Einstein, E.B., Patterson, C.A., Hon, B.J., Regan, K.A., Reddi, J., Melnikoff, D.E., Mateer, M.J., Schulz, S., Johnson, B.N., and Tallent, M.K. (2010). Somatostatin

5 signaling in neuronal cilia is critical for object recognition memory. The Journal of Neuroscience 30, 4306-4314.

Feng, X., Degese, M., Iglesias-Bartolome, R., Vaque, J.P., Molinolo, A.A., Rodrigues, M., Zaidi, R.M., Ksander, B.R., Merlino, G., Sodhi, A., et al. (2014). Hippo-independent activation of YAP by the GNAQ uveal melanoma oncogene through a Trio-regulated Rho GTPase signaling circuitry. Cancer Cell 25, 831-845.

Flatau, G., Lemichez, E., Gauthier, M., Chardin, P., Paris, S., Fiorentini, C., and Boquet, P. (1997). Toxin-induced activation of the $G$ protein $p 21$ Rho by deamidation of glutamine. Nature 387, 729-733.

Flock, T., Hauser, A.S., Lund, N., Gloriam, D.E., Balaji, S., and Babu, M.M. (2017).

Selectivity determinants of GPCR-G-protein binding. Nature 545, 317-322.

Fukata, Y., Oshiro, N., Kinoshita, N., Kawano, Y., Matsuoka, Y., Bennett, V., Matsuura, Y., and Kaibuchi, K. (1999). Phosphorylation of adducin by Rho-kinase plays a crucial role in cell motility. J Cell Biology 145, 347-361.

Gao, R., Asano, S.M., Upadhyayula, S., Pisarev, I., Milkie, D.E., Liu, T.-L., Singh, V.,

20 Graves, A., Huynh, G.H., Zhao, Y., et al. (2019). Cortical column and whole-brain imaging with molecular contrast and nanoscale resolution. Science 363, eaau8302.

Goedhart, J. (2020). PlotTwist: A web app for plotting and annotating continuous data. Plos Biol 18, e3000581.

Goetz, S.C., and Anderson, K.V. (2010). The primary cilium: a signalling centre during vertebrate development. Nature Reviews Genetics 11, 331-344.

Grimm, J.B., English, B.P., Chen, J., Slaughter, J.P., Zhang, Z., Revyakin, A., Patel, R., Macklin, J.J., Normanno, D., Singer, R.H., et al. (2015). A general method to improve fluorophores for live-cell and single-molecule microscopy. Nature Methods 12, 244-250.

Guemez-Gamboa, A., Coufal, N.G., and Gleeson, J.G. (2014). Primary cilia in the 30 developing and mature brain. Neuron 82, 511-521.

Hagen, V., Dekowski, B., Kotzur, N., Lechler, R., Wiesner, B., Briand, B., and Beyermann, M. (2008). \{7-[Bis(carboxymethyl)amino]coumarin-4-yl\}methoxycarbonyl derivatives for photorelease of carboxylic acids, alcohols/phenols, thioalcohols/thiophenols, and amines. Chem - European J 14, 1621-1627.

35 Han, B., Zhou, R., Xia, C., and Zhuang, X. (2017). Structural organization of the actinspectrin-based membrane skeleton in dendrites and soma of neurons. Proc National Acad Sci 114, E6678-E6685. 
Hansen, J.N., Rassmann, S., Stüven, B., Jurisch-Yaksi, N., and Wachten, D. (2021). CiliaQ: a simple, open-source software for automated quantification of ciliary morphology and fluorescence in 2D, 3D, and 4D images. European Phys J E 44, 18. Hilgendorf, K.I., Johnson, C.T., and Jackson, P.K. (2016). The primary cilium as a cellular receiver: organizing ciliary GPCR signaling. Curr Opin Cell Biol 39, 84-92.

Hilgendorf, K.I., Johnson, C.T., Mezger, A., Rice, S.L., Norris, A.M., Demeter, J., Greenleaf, W.J., Reiter, J.F., Kopinke, D., and Jackson, P.K. (2019). Omega-3 fatty acids activate ciliary FFAR4 to control adipogenesis. Cell 179, 1289-1305.e21.

Hirst, W.D., Minton, J.A.L., Bromidge, S.M., Moss, S.F., Latter, A.J., Riley, G., 10 Routledge, C., Middlemiss, D.N., and Price, G.W. (2000). Characterization of [125I]-SB258585 binding to human recombinant and native 5-HT6 receptors in rat, pig and human brain tissue. Brit J Pharmacol 130, 1597-1605.

Ho, J., Tumkaya, T., Aryal, S., Choi, H., and Claridge-Chang, A. (2019). Moving beyond P values: data analysis with estimation graphics. Nat Methods 16, 565-566.

15 Hodge, R.G., and Ridley, A.J. (2016). Regulating Rho GTPases and their regulators. Nat Rev Mol Cell Bio 17, 496-510.

Hoffman, D.P., Shtengel, G., Xu, C.S., Campbell, K.R., Freeman, M., Wang, L., Milkie, D.E., Pasolli, H.A., Iyer, N., Bogovic, J.A., et al. (2020). Correlative three-dimensional super-resolution and block-face electron microscopy of whole vitreously frozen cells.

20 Science 367 , eaaz5357.

Hor, C.N., Yeung, J., Jan, M., Emmenegger, Y., Hubbard, J., Xenarios, I., Naef, F., and Franken, P. (2019). Sleep-wake-driven and circadian contributions to daily rhythms in gene expression and chromatin accessibility in the murine cortex. Proc National Acad Sci 116, 25773-25783.

25 loannou, M.S., Jackson, J., Sheu, S.-H., Chang, C.-L., Weigel, A.V., Liu, H., Pasolli, H.A., Xu, C.S., Pang, S., Matthies, D., et al. (2019). Neuron-astrocyte metabolic coupling protects against activity-induced fatty acid toxicity. Cell 177, 1522-1535.e14.

Jassal, B., Matthews, L., Viteri, G., Gong, C., Lorente, P., Fabregat, A., Sidiropoulos, K., Cook, J., Gillespie, M., Haw, R., et al. (2019). The reactome pathway knowledgebase. Nucleic Acids Res 48, D498-D503.

Jiang, J.Y., Falcone, J.L., Curci, S., and Hofer, A.M. (2019). Direct visualization of cAMP signaling in primary cilia reveals up-regulation of ciliary GPCR activity following Hedgehog activation. Proc National Acad Sci, 116, 12066-12071.

Jovasevic, V., Zhang, H., Sananbenesi, F., Guedea, A.L., Soman, K.V., Wiktorowicz, 35 J.E., Fischer, A., and Radulovic, J. (2021). Primary cilia are required for the persistence of memory and stabilization of perineuronal nets. iScience 24, 102617. 
Kasthuri, N., Hayworth, K.J., Berger, D.R., Schalek, R.L., Conchello, J.A.A., KnowlesBarley, S., Lee, D., Vázquez-Reina, A., Kaynig, V., Jones, T.R., et al. (2015). Saturated reconstruction of a volume of neocortex. Cell 162, 648-661.

Kirschen, G.W., Liu, H., Lang, T., Liang, X., Ge, S., and Xiong, Q. (2017). The radial organization of neuronal primary cilia is acutely disrupted by seizure and ischemic brain injury. Frontiers in Biology 12, 124-138.

Klapoetke, N.C., Murata, Y., Kim, S., Pulver, S.R., Birdsey-Benson, A., Cho, Y., Morimoto, T.K., Chuong, A.S., Carpenter, E.J., Tian, Z., et al. (2014). Independent optical excitation of distinct neural populations. Nature Methods 11, 338-346.

10 Kohli, P., Höhne, M., Jüngst, C., Bertsch, S., Ebert, L.K., Schauss, A.C., Benzing, T., Rinschen, M.M., and Schermer, B. (2017). The ciliary membrane-associated proteome reveals actin-binding proteins as key components of cilia. Embo Rep 18, 1521-1535.

Korogod, N., Petersen, C.C., and Knott, G.W. (2015). Ultrastructural analysis of adult mouse neocortex comparing aldehyde perfusion with cryo fixation. ELife 4, e05793.

15 Lehmenkühler, A., Syková, E., Svoboda, J., Zilles, K., and Nicholson, C. (1993). Extracellular space parameters in the rat neocortex and subcortical white matter during postnatal development determined by diffusion analysis. Neuroscience 55, 339-351.

Li, Q., Lex, R.K., Chung, H., Giovanetti, S.M., Ji, Z., Ji, H., Person, M.D., Kim, J., and Vokes, S.A. (2016). The pluripotency factor NANOG binds to GLI proteins and

20 represses Hedgehog-mediated transcription. The Journal of Biological Chemistry 291, 7171-7182.

Liu, C.-M., Hsu, W.-H., Lin, W.-Y., and Chen, H.-C. (2017). Adducin family proteins possess different nuclear export potentials. J Biomed Sci 24, 30.

Lowe, D.G. (2004). Distinctive image features from scale-invariant keypoints. Int J Comput Vision 60, 91-110.

Mahlandt, E.K., Arts, J.J.G., Meer, W.J. van der, Linden, F.H. van der, Tol, S., Buul, J.D. van, Gadella, T.W.J., and Goedhart, J. (2021). Visualizing endogenous RhoA activity with an improved localization-based, genetically encoded biosensor. Biorxiv 2021.02.08.430250.

30 Masuho, I., Ostrovskaya, O., Kramer, G.M., Jones, C.D., Xie, K., and Martemyanov, K.A. (2015). Distinct profiles of functional discrimination among $G$ proteins determine the actions of G protein-coupled receptors. Sci Signal 8, ra123-ra123.

Masyuk, A.I., Huang, B.Q., Radtke, B.N., Gajdos, G.B., Splinter, P.L., Masyuk, T.V., Gradilone, S.A., and LaRusso, N.F. (2013). Ciliary subcellular localization of TGR5

35 determines the cholangiocyte functional response to bile acid signaling. American Journal of Physiology-Gastrointestinal and Liver Physiology 304, G1013-G1024. 
McLean, C.Y., Bristor, D., Hiller, M., Clarke, S.L., Schaar, B.T., Lowe, C.B., Wenger, A.M., and Bejerano, G. (2010). GREAT improves functional interpretation of cisregulatory regions. Nat Biotechnol 28, 495-501.

Meffre, J., Chaumont-Dubel, S., Cour, C. la, Loiseau, F., Watson, D.J., Dekeyne, A.,

5 Séveno, M., Rivet, J., Gaven, F., Déléris, P., et al. (2012). 5-HT6 receptor recruitment of $\mathrm{mTOR}$ as a mechanism for perturbed cognition in schizophrenia. EMBO Molecular Medicine 4, 1043-1056.

Mirvis, M., Siemers, K.A., Nelson, J.W., and Stearns, T.P. (2019). Primary cilium loss in mammalian cells occurs predominantly by whole-cilium shedding. PLOS Biology 17, e3000381.

Monsma, F., Shen, Y., Ward, R., Hamblin, M., and Sibley, D. (1993). Cloning and expression of a novel serotonin receptor with high affinity for tricyclic psychotropic drugs. Molecular Pharmacology 43, 320-327.

Mullins, N., Forstner, A.J., O’Connell, K.S., Coombes, B., Coleman, J.R.I., Qiao, Z., Als, 15 T.D., Bigdeli, T.B., Børte, S., Bryois, J., et al. (2021). Genome-wide association study of more than 40,000 bipolar disorder cases provides new insights into the underlying biology. Nat Genet 53, 817-829.

Muzerelle, A., Scotto-Lomassese, S., Bernard, J., Soiza-Reilly, M., and Gaspar, P. (2016). Conditional anterograde tracing reveals distinct targeting of individual serotonin cell groups (B5-B9) to the forebrain and brainstem. Brain Structure and Function 221, 535-561.

Nadim, W., Chaumont-Dubel, S., Madouri, F., Cobret, L., Tauzia, M.-L., Zajdel, P., Bénédetti, H., Marin, P., and Morisset-Lopez, S. (2016). Physical interaction between neurofibromin and serotonin 5-HT6 receptor promotes receptor constitutive activity. Proc National Acad Sci 113, 12310-12315.

Nagai, Y., Miyakawa, N., Takuwa, H., Hori, Y., Oyama, K., Ji, B., Takahashi, M., Huang, X.-P., Slocum, S.T., DiBerto, J.F., et al. (2020). Deschloroclozapine, a potent and selective chemogenetic actuator enables rapid neuronal and behavioral modulations in mice and monkeys. Nat Neurosci 23, 1157-1167.

30 Nager, A.R., Goldstein, J.S., Herranz-Pérez, V., Portran, D., Ye, F., Garcia-Verdugo, J., and Nachury, M.V. (2017). An actin network dispatches ciliary GPCRs into extracellular vesicles to modulate signaling. Cell 168, 252-263.e14.

Nishimura, A., Kitano, K., Takasaki, J., Taniguchi, M., Mizuno, N., Tago, K., Hakoshima, T., and Itoh, H. (2010). Structural basis for the specific inhibition of heterotrimeric $\mathrm{Gq}$ protein by a small molecule. Proc National Acad Sci 107, 13666-13671.

Oikonomou, G., Altermatt, M., Zhang, R., Coughlin, G.M., Montz, C., Gradinaru, V., and Prober, D.A. (2019). The serotonergic raphe promote sleep in zebrafish and mice. Neuron 103, 686-701.e8. 
Okashah, N., Wan, Q., Ghosh, S., Sandhu, M., Inoue, A., Vaidehi, N., and Lambert, N.A. (2019). Variable $G$ protein determinants of GPCR coupling selectivity. Proc National Acad Sci 116, 12054-12059.

Pan, L., Yan, R., Li, W., and Xu, K. (2018). Super-resolution microscopy reveals the native ultrastructure of the erythrocyte cytoskeleton. Cell Reports 22, 1151-1158.

Park, Y.-G., Sohn, C., Chen, R., McCue, M., Yun, D., Drummond, G.T., Ku, T., Evans, N.B., Oak, H., Trieu, W., et al. (2018). Protection of tissue physicochemical properties using polyfunctional crosslinkers. Nature Biotechnology 37, 73-83.

Plessner, M., and Grosse, R. (2019). Dynamizing nuclear actin filaments. Curr Opin Cell Biol 56, 1-6.

Postma, M., and Goedhart, J. (2019). PlotsOfData - A web app for visualizing data together with their summaries. Plos Biol 17, e3000202.

Püspöki, Z., Storath, M., Sage, D., and Unser, M. (2016). Transforms and operators for directional bioimage analysis: a survey. Adv Anat Embryology Cell Biology 219, 69-93.

15 Rahman, Md., Kim, H., Lee, K., Yun, H.-M., Hong, J.-H., Kim, Y., Choo, H., Park, M., and Rhim, H. (2017). 5-hydroxytryptamine 6 receptor (5-HT6R)-mediated morphological changes via RhoA-dependent pathways. Molecules and Cells 40, 495-502.

Rasch, B., and Born, J. (2013). About sleep's role in memory. Physiol Rev 93, 681-766.

Raschka, S. (2018). MLxtend: Providing machine learning and data science utilities and extensions to Python's scientific computing stack. JOSS 3, 638.

Raudvere, U., Kolberg, L., Kuzmin, I., Arak, T., Adler, P., Peterson, H., and Vilo, J. (2019). g:Profiler: a web server for functional enrichment analysis and conversions of gene lists (2019 update). Nucleic Acids Res 47, W191-W198.

Rezakhaniha, R., Agianniotis, A., Schrauwen, J.T.C., Griffa, A., Sage, D., Bouten, 25 C.V.C., Vosse, F.N. van de, Unser, M., and Stergiopulos, N. (2012). Experimental investigation of collagen waviness and orientation in the arterial adventitia using confocal laser scanning microscopy. Biomech Model Mechan 11, 461-473.

Rolf, H., Olga, K., Sravasti, M., Jeffrey, K., Bram, van den B., and Kees, J. (2021). Dynamic FRET-FLIM based screens of signal transduction pathways: a feasibility study. 30 Biorxiv 2021.05.23.445328.

Schechter, L.E., Lin, Q., Smith, D.L., Zhang, G., Shan, Q., Platt, B., Brandt, M.R., Dawson, L.A., Cole, D., Bernotas, R., et al. (2007). Neuropharmacological profile of novel and selective 5-HT6 receptor agonists: WAY-181187 and WAY-208466. Neuropsychopharmacology 33, 1323-1335. 
Schmidt, G., Sehr, P., Wilm, M., Selzer, J., Mann, M., and Aktories, K. (1997). GIn 63 of Rho is deamidated by Escherichia coli cytotoxic necrotizing factor-1. Nature 387, 725729.

Schoenfelder, S., and Fraser, P. (2019). Long-range enhancer-promoter contacts in gene expression control. Nat Rev Genet 20, 437-455.

Seifert, R., and Wenzel-Seifert, K. (2002). Constitutive activity of G-protein-coupled receptors: cause of disease and common property of wild-type receptors. NaunynSchmiedeberg's Archives Pharmacol 366, 381-416.

Sen, B., Uzer, G., Samsonraj, R.M., Xie, Z., McGrath, C., Styner, M., Dudakovic, A., 10 Wijnen, A.J. van, and Rubin, J. (2017). Intranuclear actin structure modulates mesenchymal stem cell differentiation. Stem Cells 35, 1624-1635.

Sosinsky, G.E., Crum, J., Jones, Y.Z., Lanman, J., Smarr, B., Terada, M., Martone, M.E., Deerinck, T.J., Johnson, J.E., and Ellisman, M.H. (2008). The combination of chemical fixation procedures with high pressure freezing and freeze substitution preserves highly labile tissue ultrastructure for electron tomography applications. Journal of Structural Biology 161, 359-371.

Spencer, V.A., Costes, S., Inman, J.L., Xu, R., Chen, J., Hendzel, M.J., and Bissell, M.J. (2010). Depletion of nuclear actin is a key mediator of quiescence in epithelial cells. J Cell Sci 124, 123-132.

20 Stringer, C., Wang, T., Michaelos, M., and Pachitariu, M. (2021). Cellpose: a generalist algorithm for cellular segmentation. Nat Methods 18, 100-106.

Stuart, T., Srivastava, A., Lareau, C., and Satija, R. (2020). Multimodal single-cell chromatin analysis with Signac. Biorxiv 2020.11.09.373613.

Sun, Z., Wang, B., Chen, C., Li, C., and Zhang, Y. (2021). 5-HT6R null mutation induces synaptic and cognitive defects. Aging Cell 20, e13369.

Takasaki, J., Saito, T., Taniguchi, M., Kawasaki, T., Moritani, Y., Hayashi, K., and Kobori, M. (2004). A novel Gaq/11-selective inhibitor. J Biol Chem 279, 47438-47445.

Tanaka, T., Nishimura, D., Wu, R.-C., Amano, M., Iso, T., Kedes, L., Nishida, H., Kaibuchi, K., and Hamamori, Y. (2006). Nuclear Rho Kinase, ROCK2, Targets p300 30 Acetyltransferase. J Biol Chem 281, 15320-15329.

Tereshko, L., Gao, Y., Cary, B.A., Turrigiano, G.G., and Sengupta, P. (2021). Ciliary neuropeptidergic signaling dynamically regulates excitatory synapses in postnatal neocortical pyramidal neurons. Elife 10, e65427.

Thomas, D., S., P., Ronald, W., F., David, Miloslav, S., Ya-Xian, W., D., B., Stephan, Shu-Hsien, S., W., G., Jeffrey, Dimitrios, K., P., M., Mark, et al. (2019). Comparing 3D ultrastructure of presynaptic and postsynaptic mitochondria. Biol Open 8, bio044834. 
Ting, J.T., Lee, B.R., Chong, P., Soler-Llavina, G., Cobbs, C., Koch, C., Zeng, H., and Lein, E. (2018). Preparation of acute brain slices using an optimized N-Methyl-Dglucamine protective recovery method. J Vis Exp Jove 53825.

Unger, E.K., Keller, J.P., Altermatt, M., Liang, R., Matsui, A., Dong, C., Hon, O.J., Yao, Z., Sun, J., Banala, S., et al. (2020). Directed evolution of a selective and sensitive serotonin sensor via machine learning. Cell 183, 1986-2002.e26.

Upton, N., Chuang, T., Hunter, A.J., and Virley, D.J. (2008). 5-HT6 receptor antagonists as novel cognitive enhancing agents for Alzheimer's disease. Neurotherapeutics 5, 458-469.

10 Wan, O.W., Shin, E., Mattsson, B., Caudal, D., Svenningsson, P., and Björklund, A. (2016). a-Synuclein induced toxicity in brain stem serotonin neurons mediated by an AAV vector driven by the tryptophan hydroxylase promoter. Sci Reports 6, 26285.

Wan, J., Peng, W., Li, X., Qian, T., Song, K., Zeng, J., Deng, F., Hao, S., Feng, J., Zhang, P., et al. (2021). A genetically encoded sensor for measuring serotonin dynamics. Nat Neurosci 24, 746-752.

Williams, S.L., Lutz, S., Charlie, N.K., Vettel, C., Ailion, M., Coco, C., Tesmer, J., Jorgensen, E.M., Wieland, T., and Miller, K.G. (2007). Trio's Rho-specific GEF domain is the missing Gaq effector in C. elegans. Genes \& Development 21, 2731-2746.

Wong, P.T., Roberts, E.W., Tang, S., Mukherjee, J., Cannon, J., Nip, A.J., Corbin, K.,

20 Krummel, M.F., and Choi, S. (2017). Control of an unusual Photo-Claisen rearrangement in coumarin caged tamoxifen through an extended spacer. ACS Chem Biol 12, 1001-1010.

Wu, Y., Whiteus, C., Xu, S.C., Hayworth, K.J., Weinberg, R.J., Hess, H.F., and Camilli, P. (2017). Contacts between the endoplasmic reticulum and other membranes in neurons. Proc National Acad Sci 114, E4859-E4867.

Xie, L., Dong, P., Chen, X., Hsieh, T.-H.S., Banala, S., Marzio, M.D., English, B.P., Qi, Y., Jung, S.K., Kieffer-Kwon, K.-R., et al. (2020). 3D ATAC-PALM: super-resolution imaging of the accessible genome. Nat Methods 17, 430-436.

Zechmann, B., Müller, M., and Zellnig, G. (2007). Membrane associated qualitative

30 differences in cell ultrastructure of chemically and high pressure cryofixed plant cells. J Struct Biol 158, 370-377.

Zeisel, A., Hochgerner, H., Lönnerberg, P., Johnsson, A., Memic, F., Zwan, J. van der, Häring, M., Braun, E., Borm, L.E., Manno, G., et al. (2018). Molecular architecture of the mouse nervous system. Cell 174, 999-1014.e22.

35 Zhang, Z., Gao, K., Liu, Q., Zhou, J., Li, X., Lang, N., Liu, M., Wang, T., Zhang, J., Wang, H., et al. (2020). Somatic variants in new candidate genes identified in focal cortical dysplasia type II. Epilepsia 61, 667-678. 
Zhao, K., Wang, W., Rando, O.J., Xue, Y., Swiderek, K., Kuo, A., and Crabtree, G.R. (1998). Rapid and phosphoinositol-dependent binding of the SWI/SNF-like BAF complex to chromatin after T lymphocyte receptor signaling. Cell 95, 625-636.

Zheng, Q., Ayala, A.X., Chung, I., Weigel, A.V., Ranjan, A., Falco, N., Grimm, J.B., 5 Tkachuk, A.N., Wu, C., Lippincott-Schwartz, J., et al. (2019). Rational design of fluorogenic and spontaneously blinking labels for super-resolution imaging. ACS Central Sci 5, 1602-1613. 


\section{$A$}

ADCY3 cilia

PCP4 CA2

B

CA1 str. oriens s.
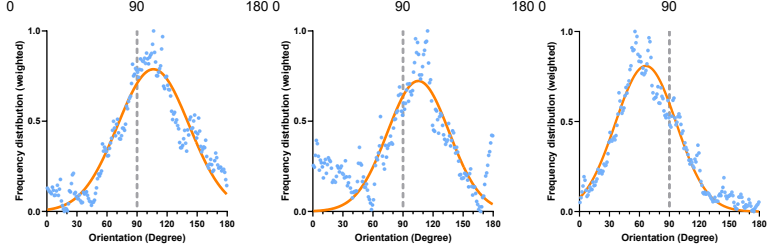

CA3 : str. oriens

\section{CA1}

str. oriens

\section{i. CA2}

\section{$\square \mathrm{CA3}$}

$500 \mu \mathrm{m}$

$\because-1 \%$ D

ADCY3 cilia str. rad.

CROCC rootlet

$20 \mu \mathrm{m}$
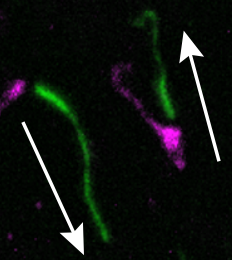

$2 x y=4$

ADCY3 cilia

CROCC rootlet

$5 \mu \mathrm{m}$

Figure 1 


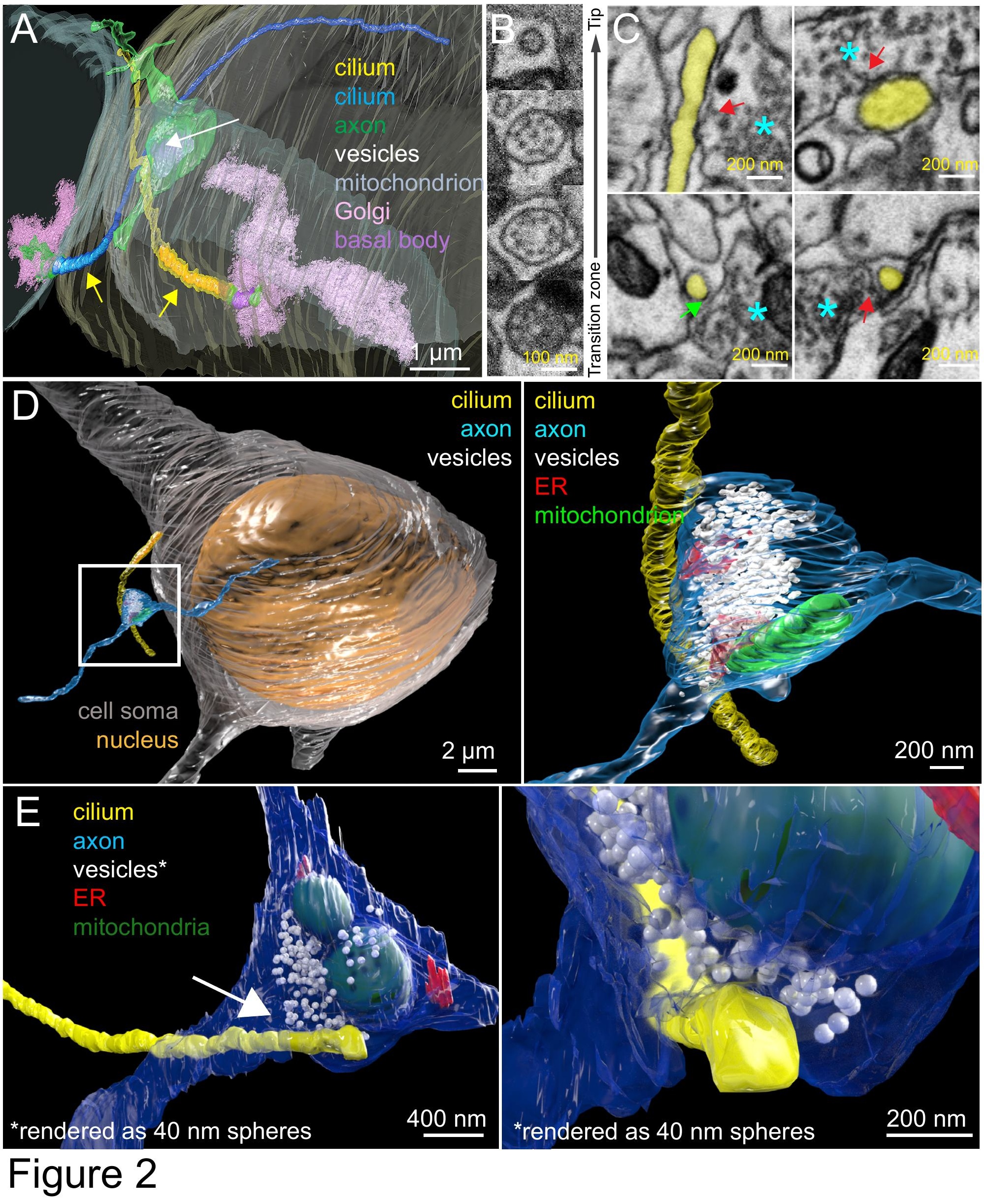


A Hoechst

ADD1

$M A D O$

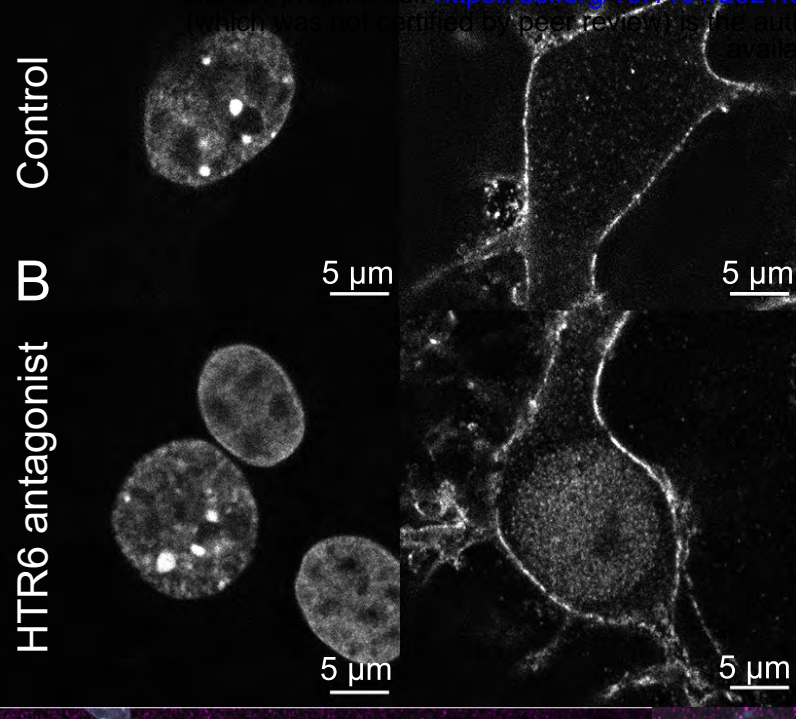

C ADD1

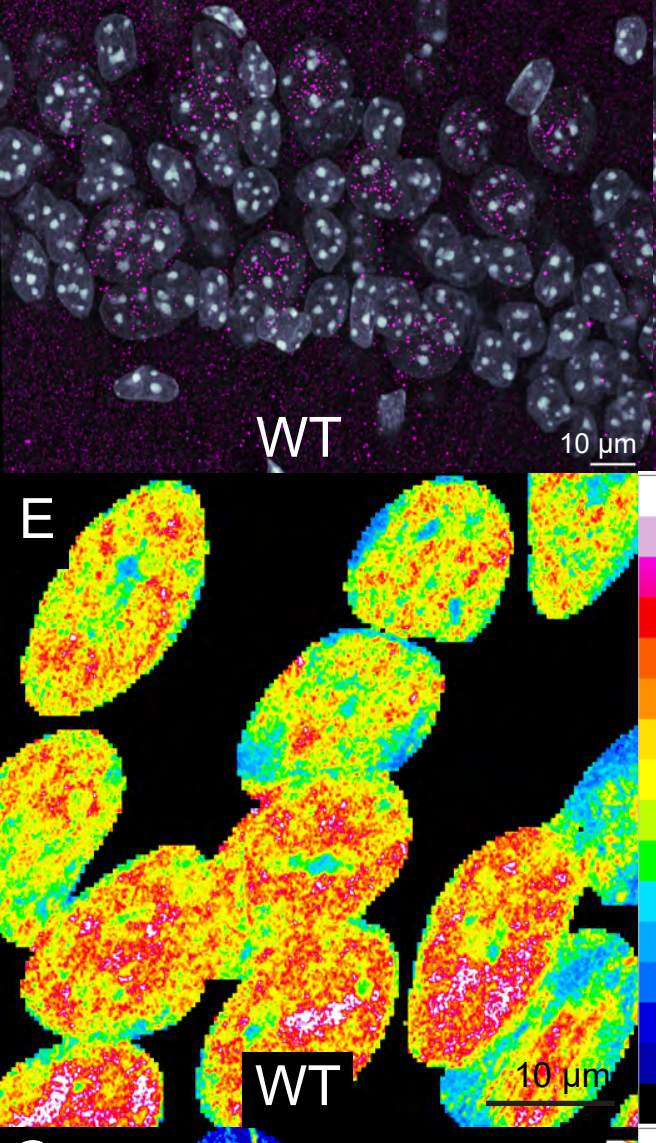

$6 \because \because$

\section{ADD1}

$\mu$
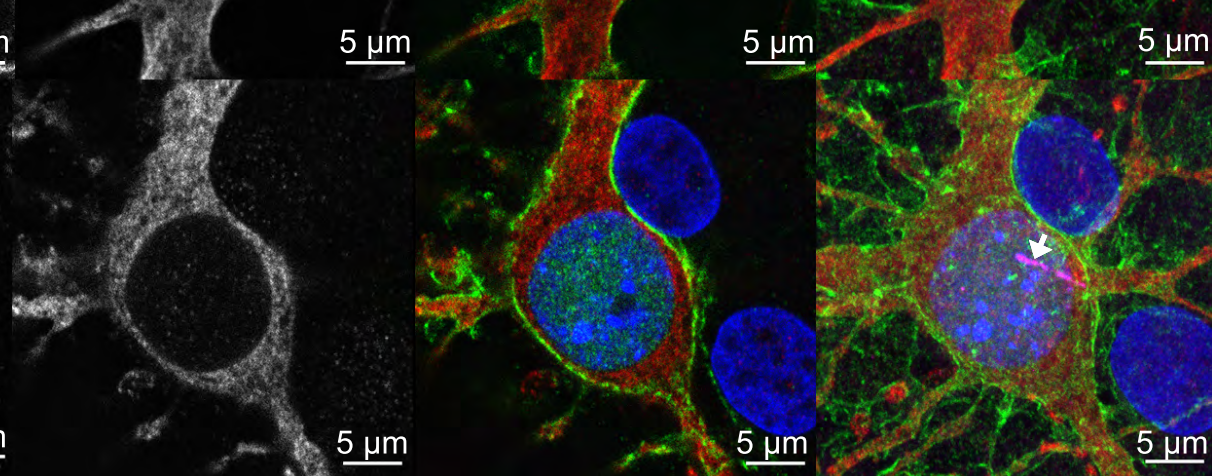

8 . D

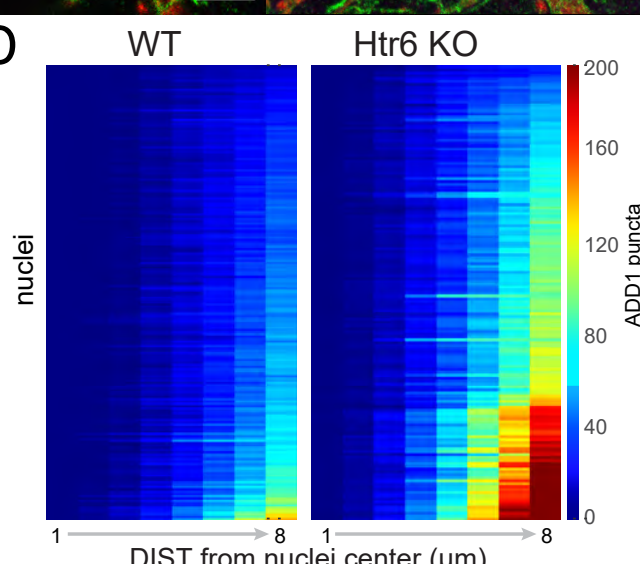

C

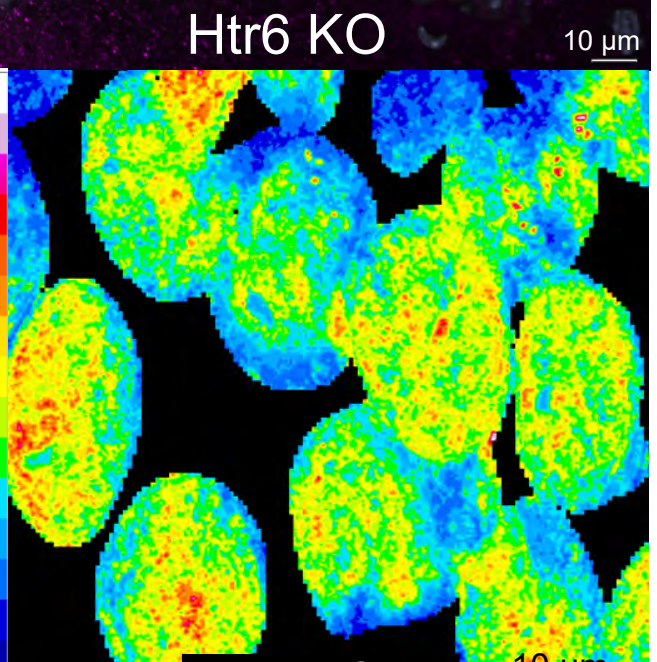

$F$

DIST from nuclei center $(\mu \mathrm{m})$

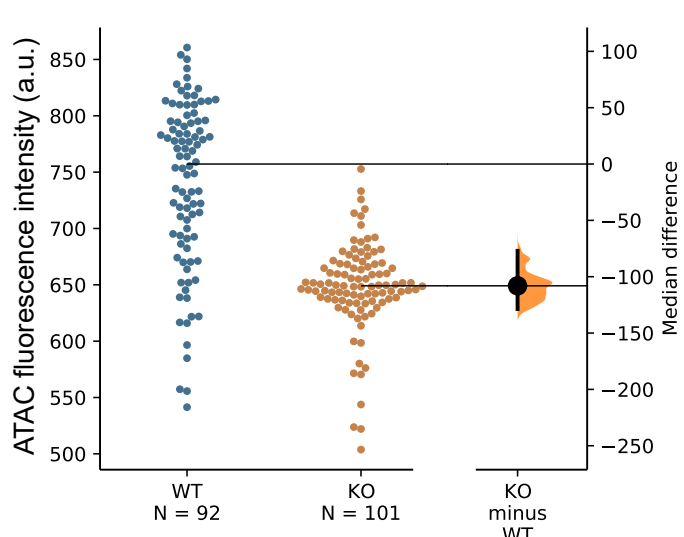

Htr6 KO

$\stackrel{2}{\longrightarrow}$

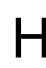

to
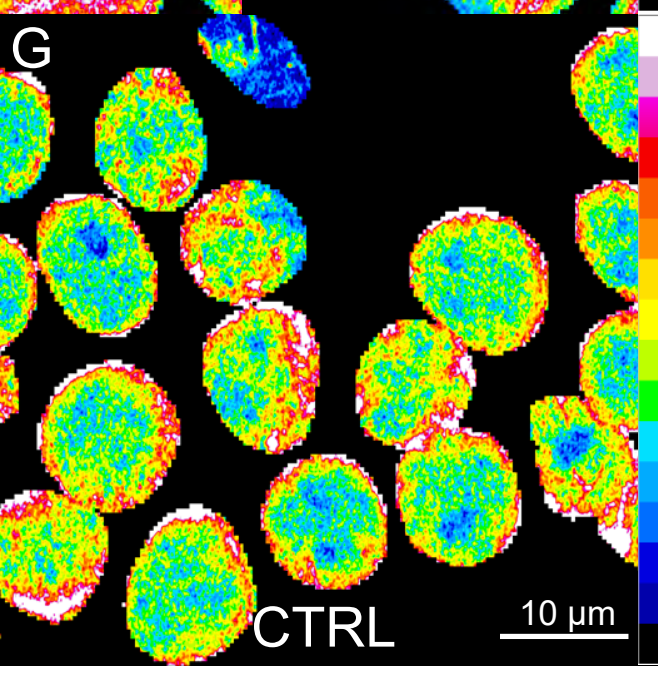
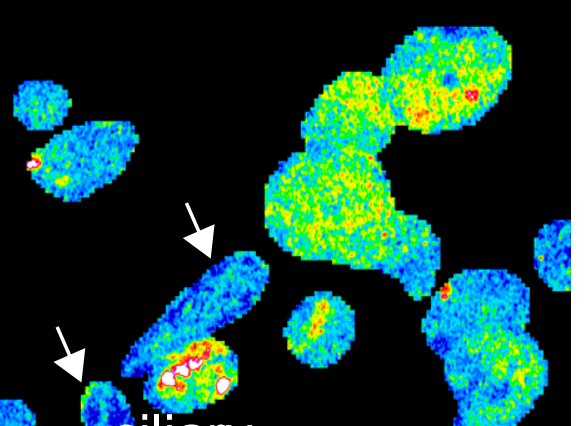
A
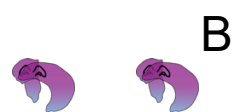

B

Wild-type Snap-frozen HTR6-Ko Nuclei isolation

10x scATAC-seq and sequencing Data processing (pooled)

Cell clustering and marker identification Cell type identification

Comparison of WT versus HTR6 KO CA1 neurons
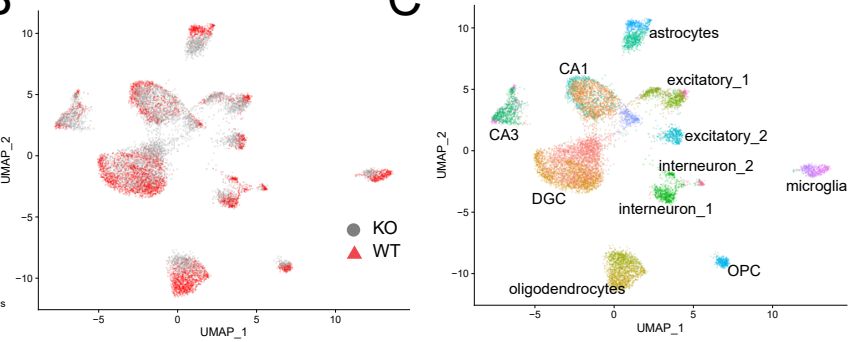

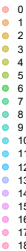

orig.ident KO

D

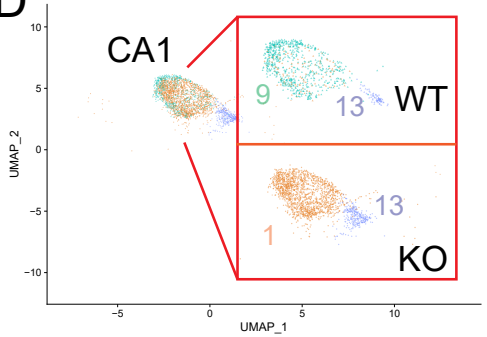

E
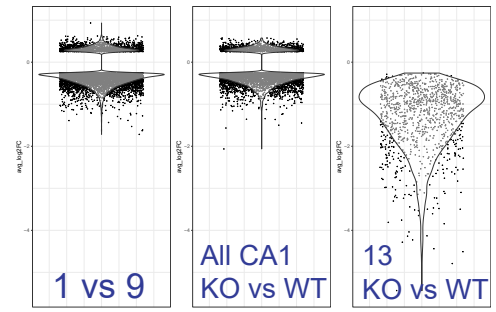

F

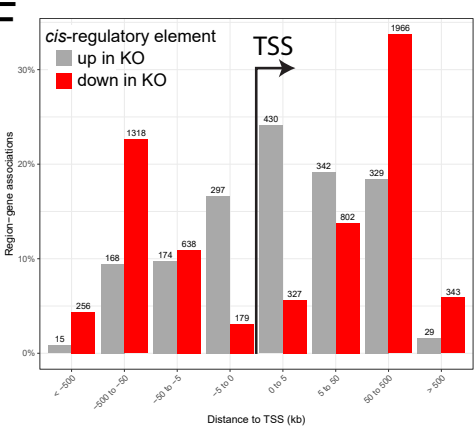

G

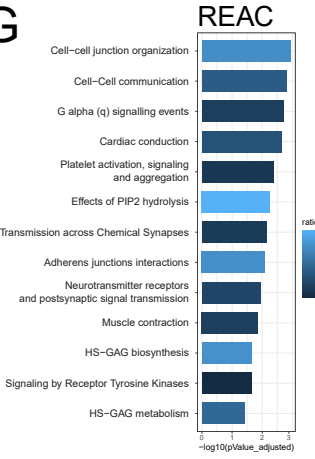

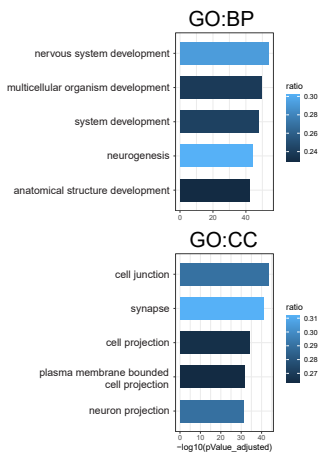

\section{Figure 7}




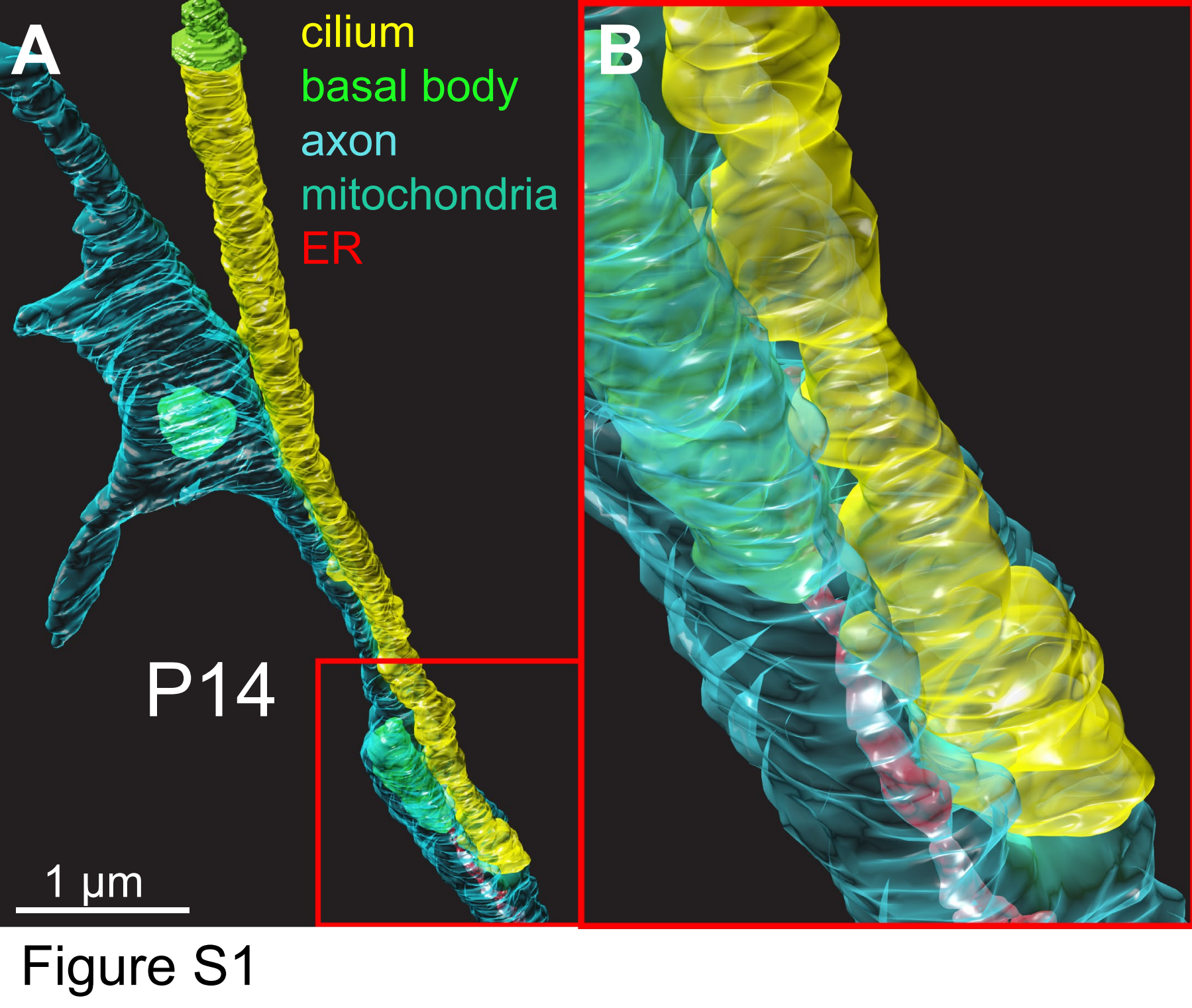




\section{Hybrid protocol}

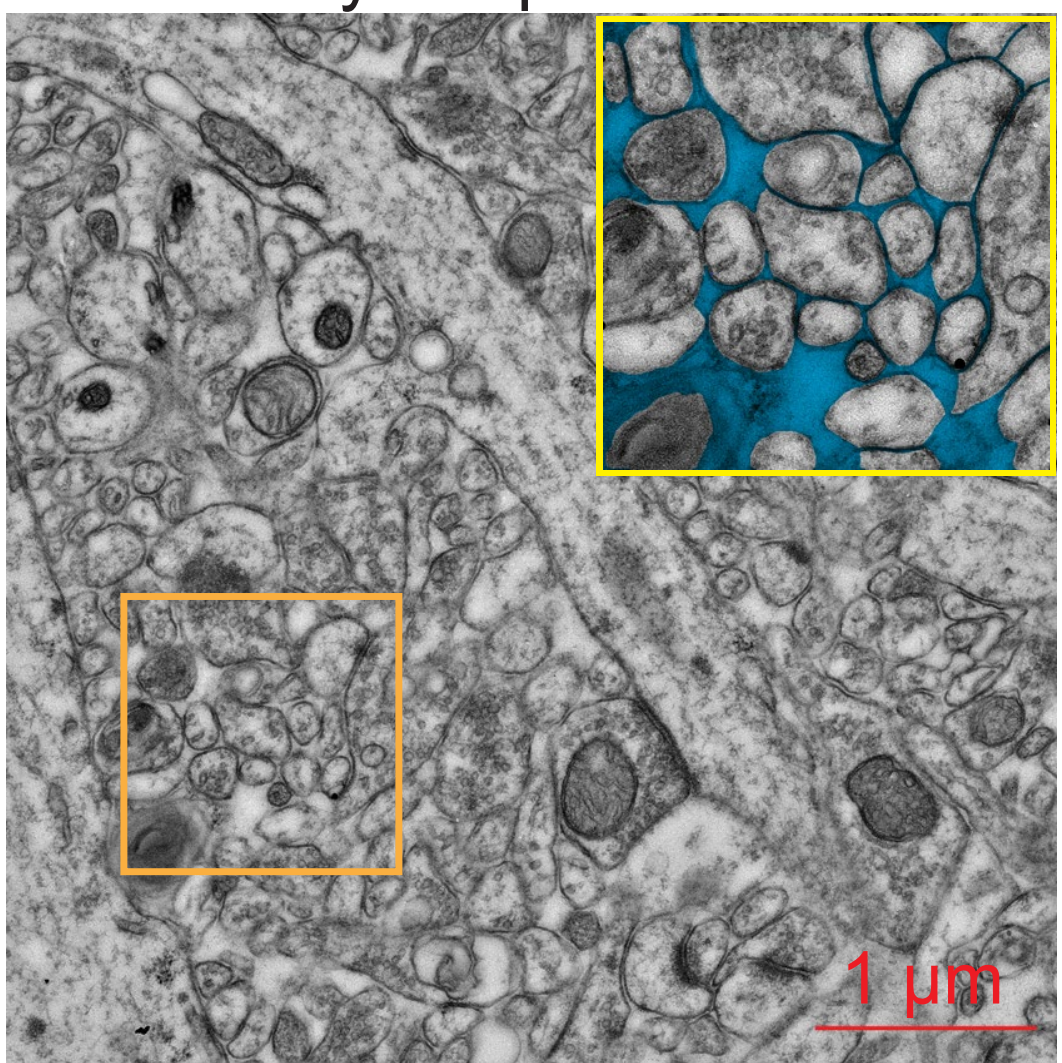

Conventional protocol

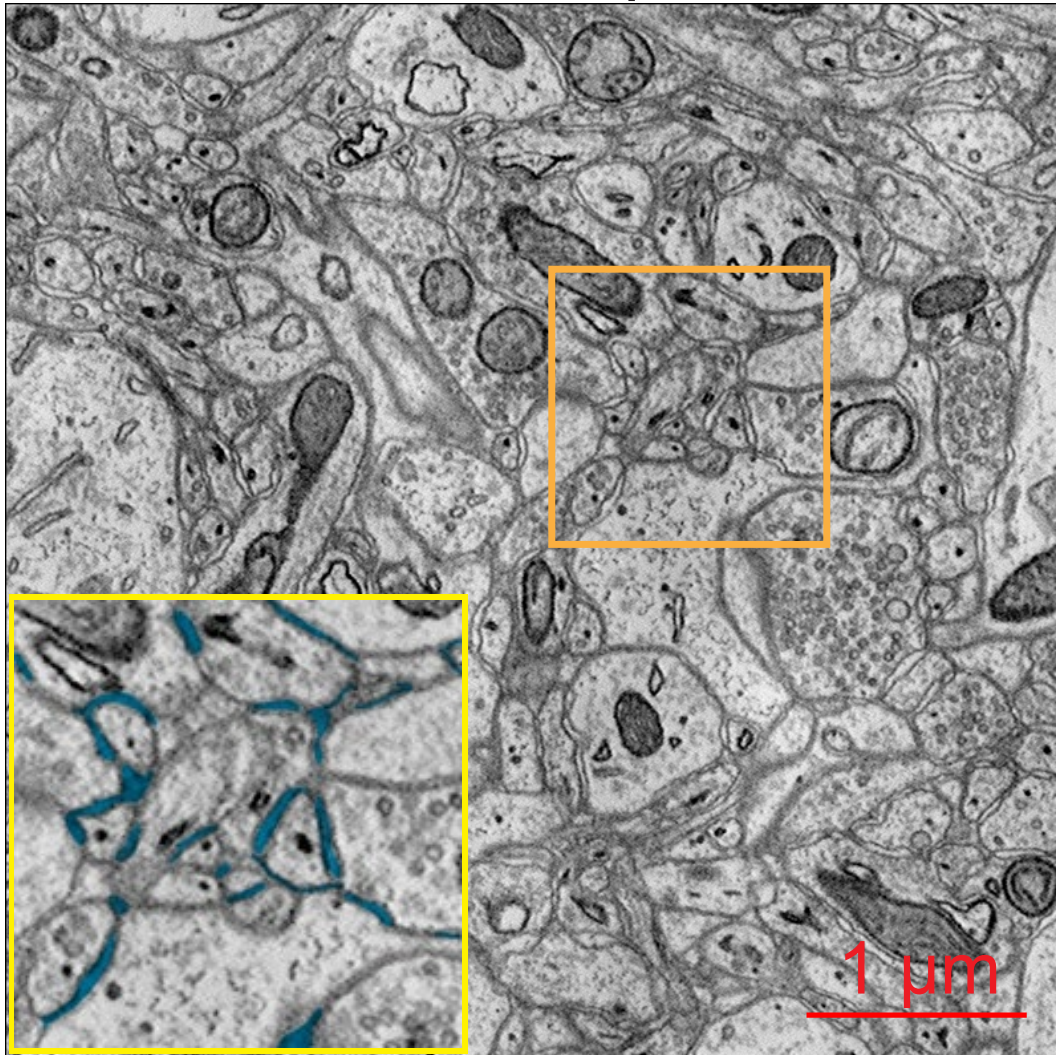

\section{Figure S2}


A

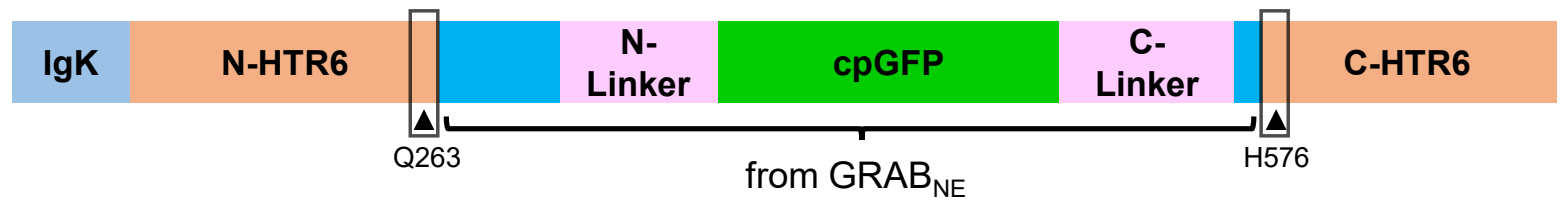

B

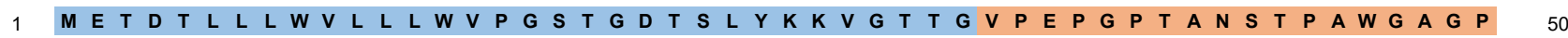

51 P S A P G G S G W V A A A L C V V I A L T A A A N S L L I A L I C

101 T S D L M V G L V V M P P A M L N A L Y G R W V L A R G L C L L W T A F D V M C C S A S I L N L C L 150

151 I S L D R Y L L I L S P L R Y K L R M T P L R A L A L V L G A W S L A A L A S F L P L L L G W H E L 200

201 G H A R P P V P G Q C R L L A S L P F V L V A S G L T F F L P

251 V $Q$ Q V A A S L T T G M A S Q

301 T Q L N G A P G E P A P A G P R D T D A L D L E E G G N V Y I K A D K Q K N G I K A N F H I R H N I 350

351 E D G G V Q L A Y H Y Q Q N T P I G D G P V L L P D N H Y L S V Q S

401 E F V T T A A G I T

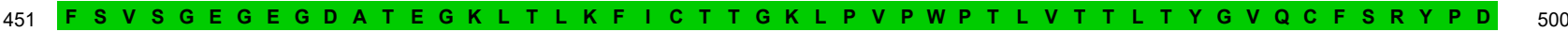

501 H M K Q H D F F K S A M P E G Y I Q E R T I F F K D D G N Y K T R A E V K F E G D T L V N R I E L K 550

551 G I D F $\quad$ K E E D G N I L G H K L E 601 F F V A N I V Q A V C D C I S P G L F D V L T W L G Y C N S T M N P I I Y P L F M R D F K R A L G R 651 F L P C P R C P R E R Q A S L A S P S L R T S H S G P R P G L S L Q Q V L P L P L P P D S D S D S D 700 701 A G S G G S S G L R L T A Q L L L P G E A T Q D P P L P T R A A A A V N F F N I D P A E P E L R P H 750 751 P L G I P T I R L A P L S L P P P *
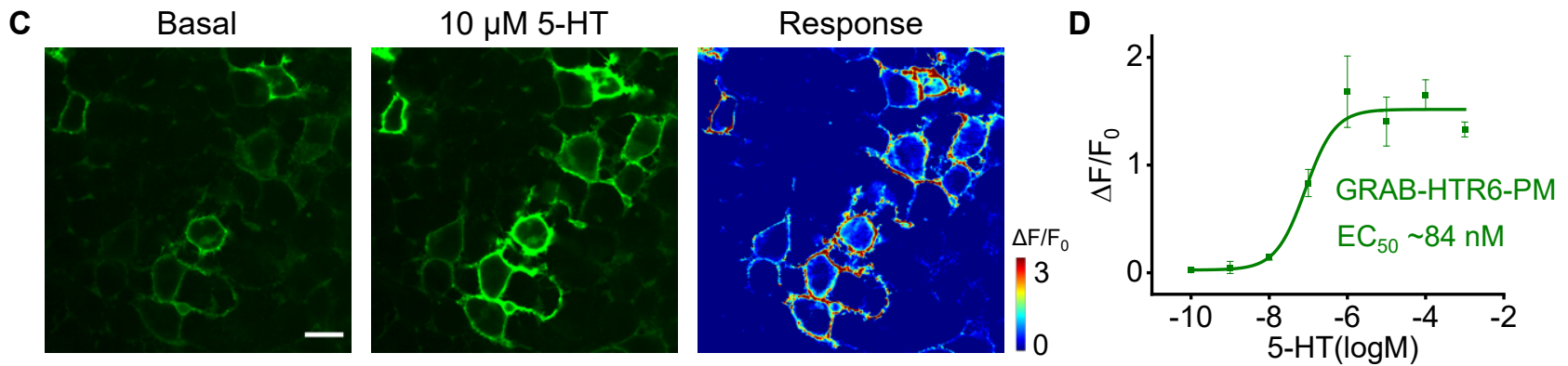

\section{Figure S3}


MAP2 pyramidal neurons SERT serotonergic axons HFR6 Halotag-JF552 cilium

hippocampus

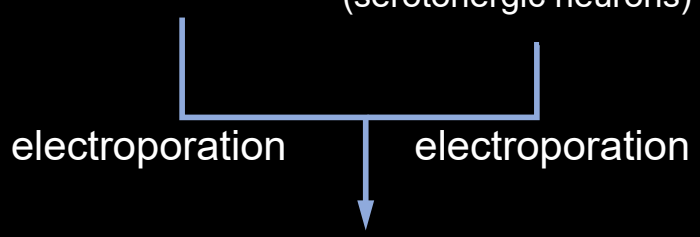

Midbrain raphe (serotonergic neurons)

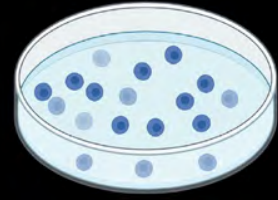

co-culture AAV transfection

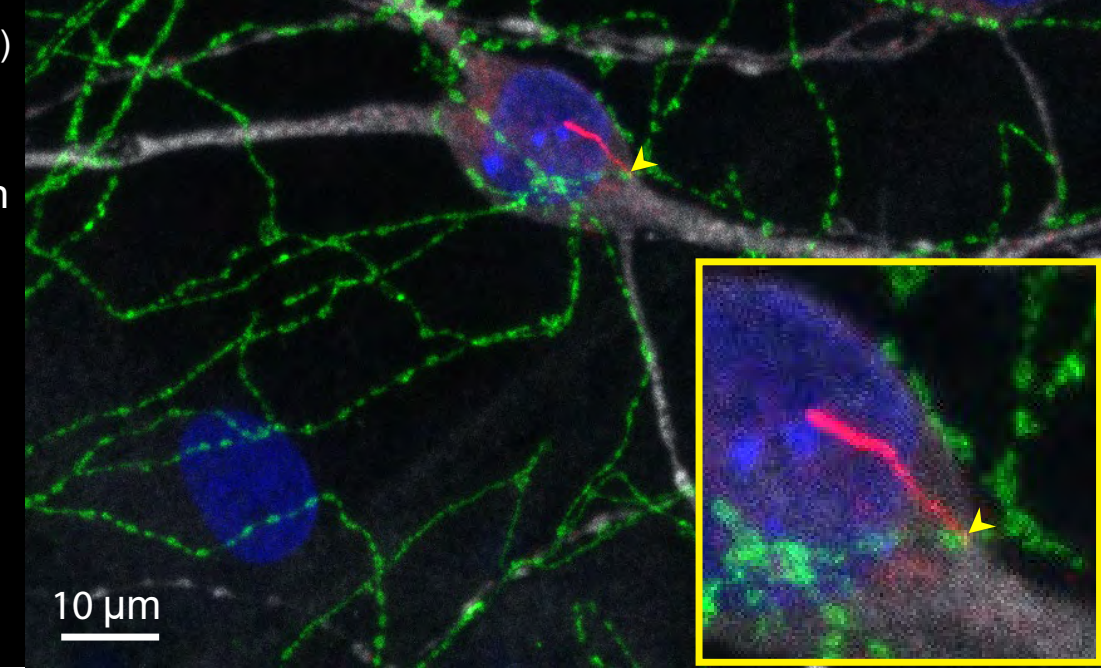

\section{Figure S4}


bioRxiv preprint doi: https://doi.org/10.1101/2021.09.27.461878; this version posted September 28, 2021. The copyright holder for this preprint

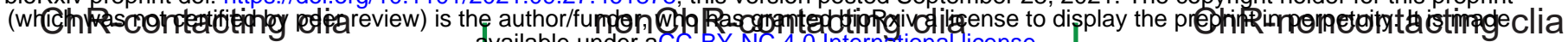

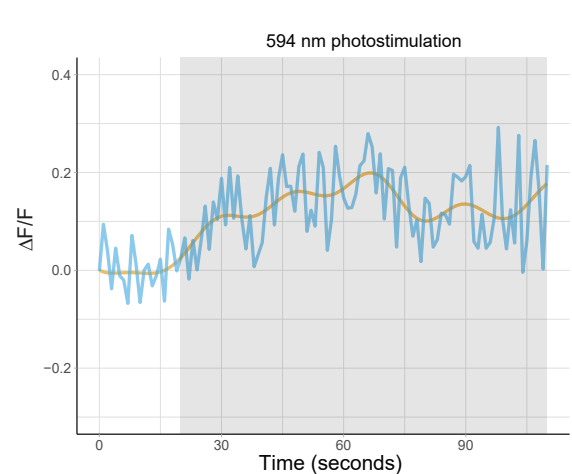
available under aCC-BY-NC 4.0 International license.
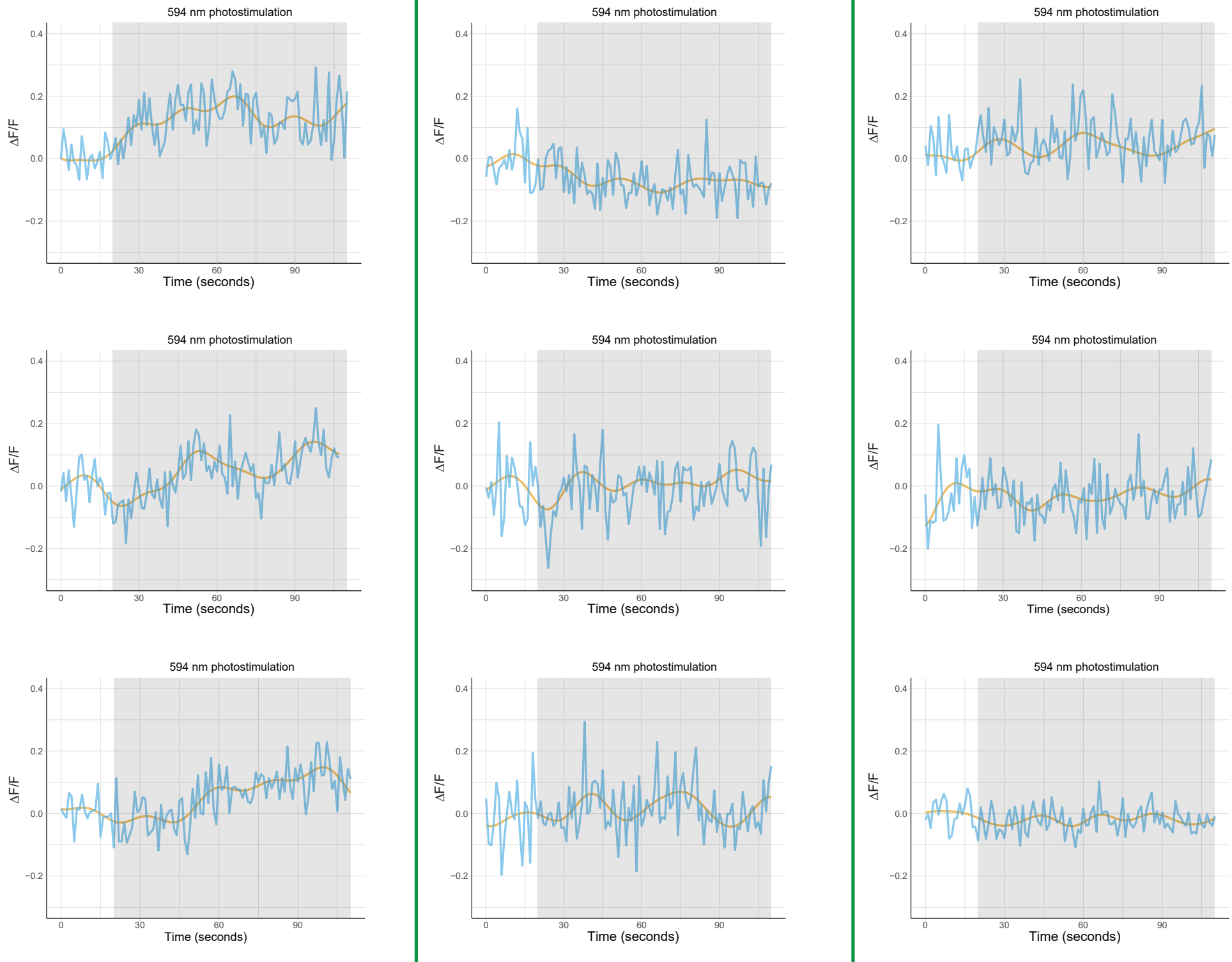

\section{Figure S5}




\section{Gene activity markers by cluster}

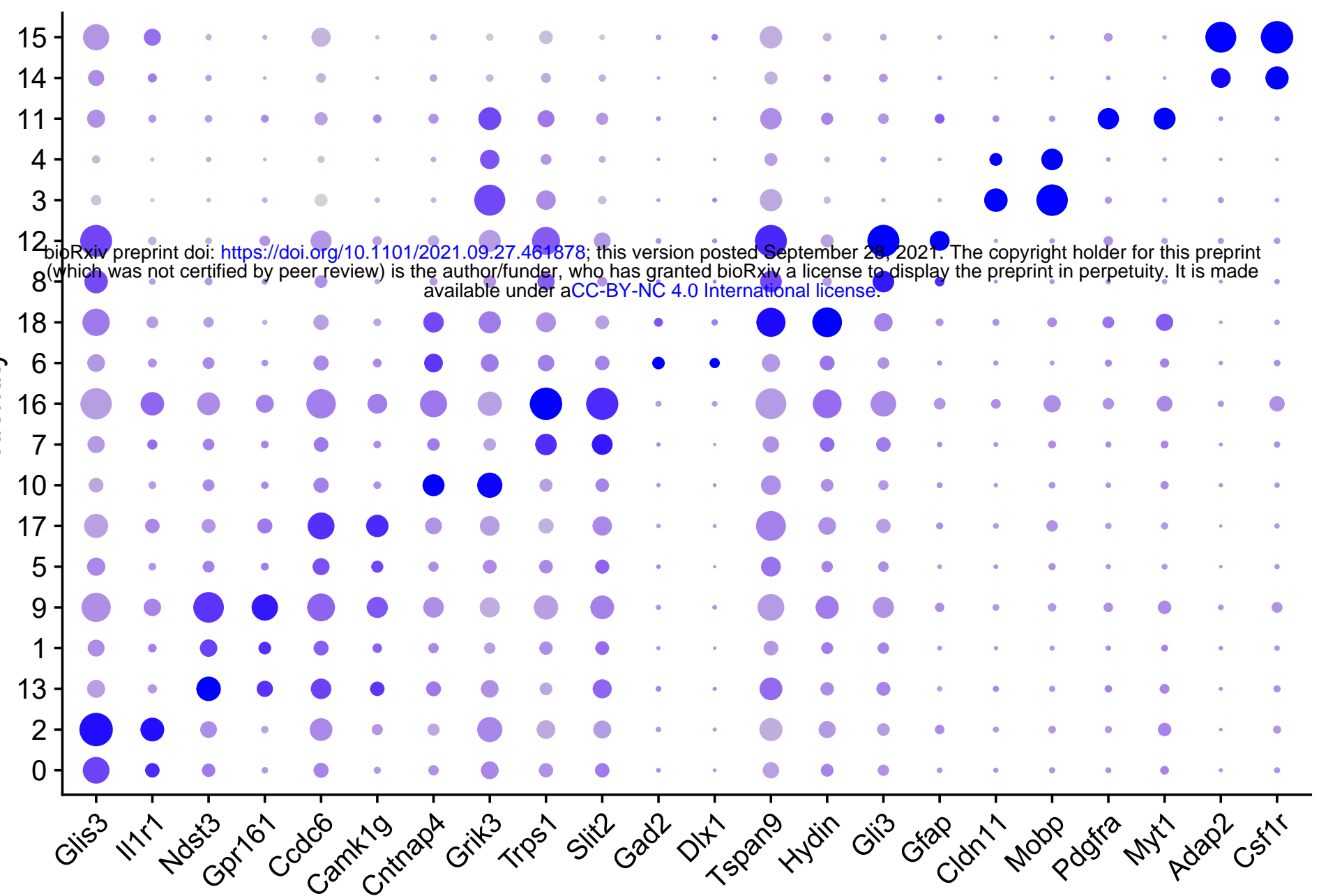

Features

\section{Gene activity markers by cell type}

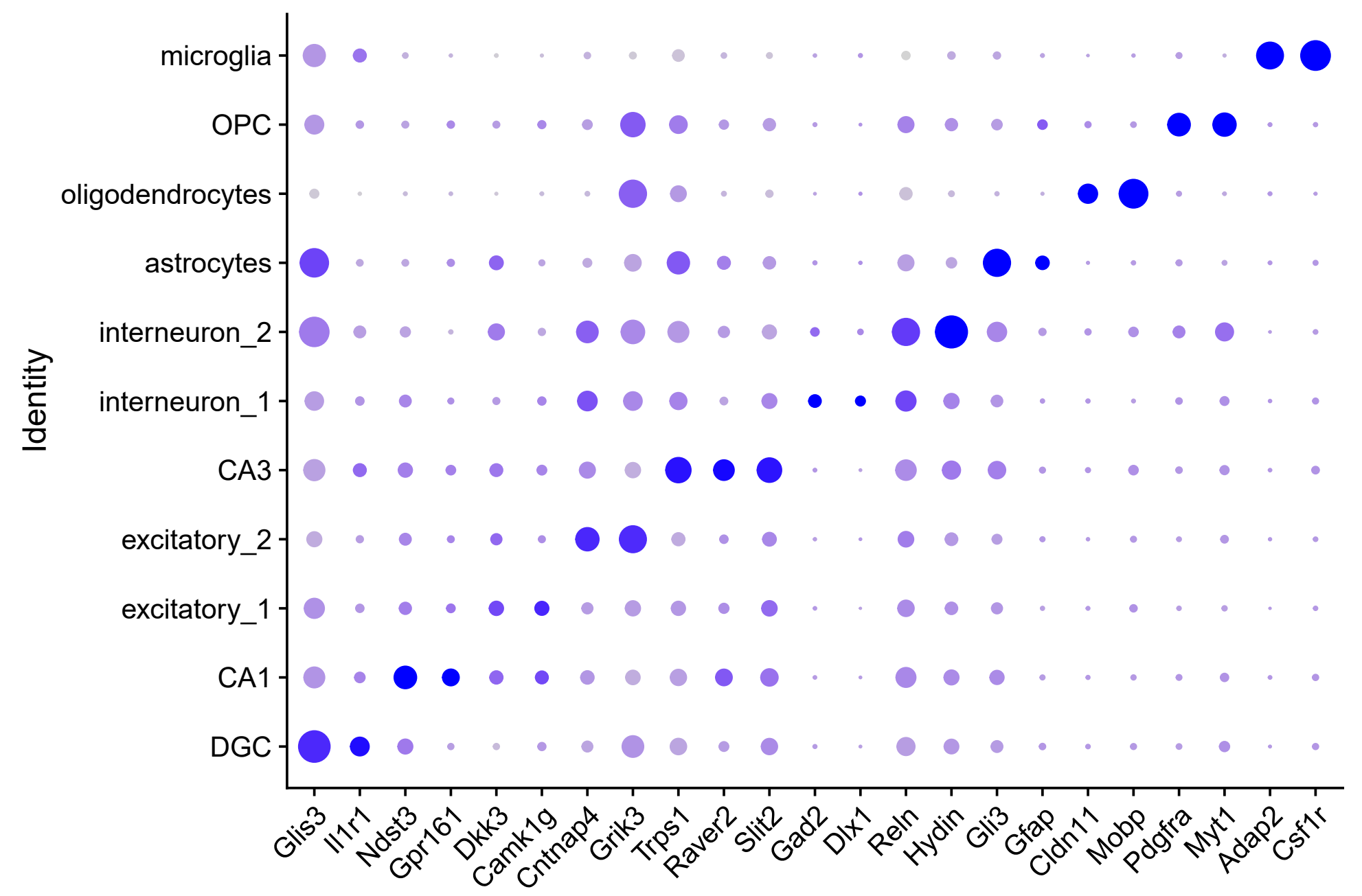

Percent Expressed
-25
50
75
Average Expression
-2
1
0
-1



Percent Expressed
-1
1
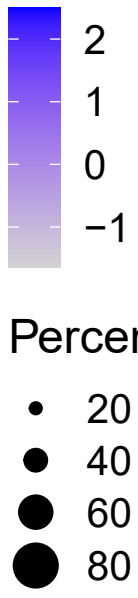

\section{Features}


